Contract Program or Project Title:

Subject of this Document:

Type of Document:

Authors:

Date of Document:

Responsible NRC Individual and NRC Office or Division
Structural Integrity Assessment Procedures for Pressurized Components in Nuclear Power Plants Program

Cleavage Fracture Modeling of Pressure Vessels under Transient Thermo-Mechanical Loading

Letter Report

X. Qian (National University of Singapore)

R. H. Dodds Jr. (University of Illinois at Urbana Champaign)

S. Yin (Oak Ridge National Laboratory)

B. R. Bass (Oak Ridge National Laboratory)

October 2007

M. T. EricksonKirk

Division of Engineering Technology

Office of Nuclear Regulatory Research

Prepared by the

Oak Ridge National Laboratory

PO Box 2008

Oak Ridge, Tennessee 37831-6085

managed by

UT-Battelle, LLC

for the

Office of Nuclear Regulatory Research

U. S. Nuclear Regulatory Commission

Washington, DC 20555-0001

under Interagency Agreement DOE 1886-N637-8Y

NRC JCN N6458 


\title{
Cleavage Fracture Modeling of Pressure Vessels under Transient Thermo-Mechanical Loading
}

\author{
Manuscript Completed— October 2007 \\ Date Published-
}

Prepared by:

X. Qian, R. H. Dodds Jr.,

S. Yin and B. R. Bass

Prepared by the

Oak Ridge National Laboratory

PO Box 2008

Oak Ridge, Tennessee 37831-6085

managed by

UT-Battelle, LLC for the

Office of Nuclear Regulatory Research

U.S. Nuclear Regulatory Commission

Washington, DC 20555-0001

under Interagency Agreement DOE 1886-N637-8Y

NRC JCN N6458 


CAUTION
This document has not been given final patent
clearance and is for internal use only. If this
document is to be given public release, it must be
cleared through the site Technical Information
Office, which will see that the proper patent and
technical information reviews are completed in
accordance with the policies of Oak Ridge
National Laboratory and UT-Battelle, LLC.

This report was prepared as an account of work sponsored by an agency of the United States government. Neither the United States government nor any agency thereof, nor any of their employees, makes any warranty, express or implied, or assumes any legal liability or responsibility for the accuracy, completeness, or usefulness of any information, apparatus, product, or process disclosed, or represents that its use would not infringe privately owned rights. Reference herein to any specific commercial product, process, or service by trade name, trademark, manufacturer, or otherwise, does not necessarily constitute or imply its endorsement, recommendation, or favoring by the United States government or any agency thereof. The views and opinions of authors expressed herein do not necessarily state or reflect those of the United States government or any agency thereof. 


\section{CONTENTS}

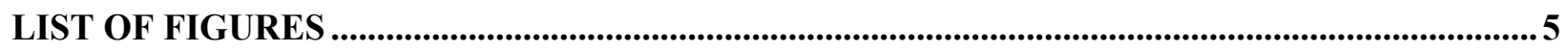

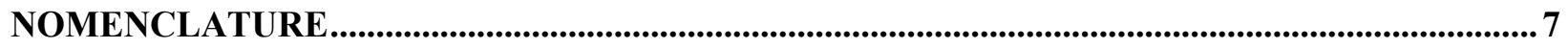

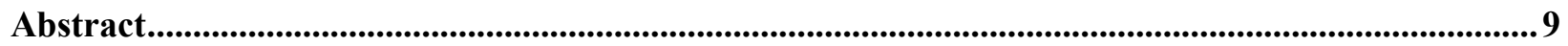

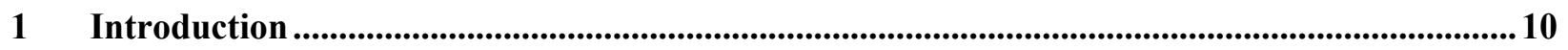

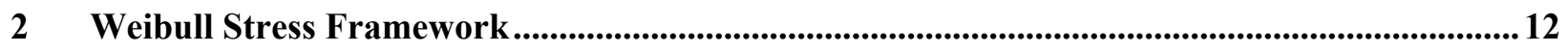

$2.1 \quad$ Weibull Stress Model............................................................................................................................. 12

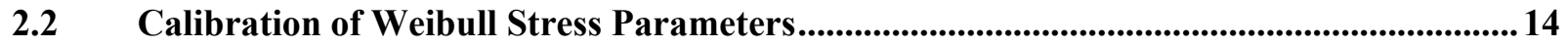

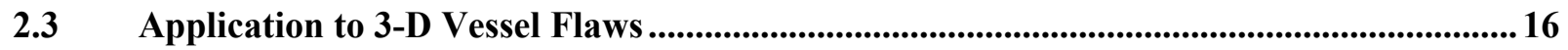

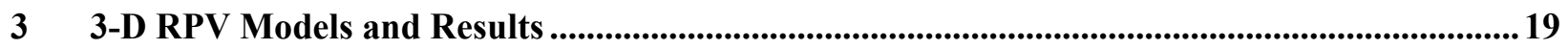

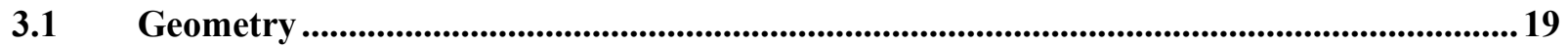

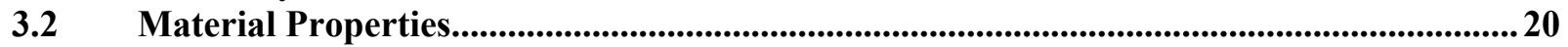

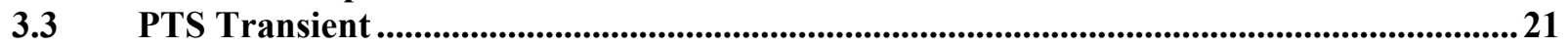

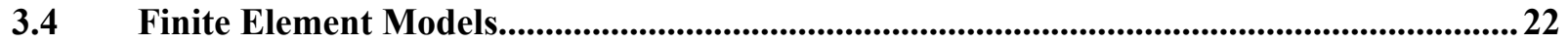

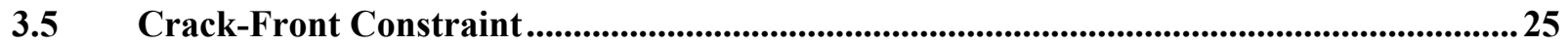

3.6 Weibull Stress Values for the 3-D RPV model........................................................................... 27

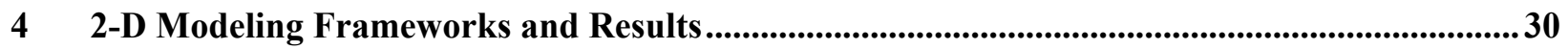

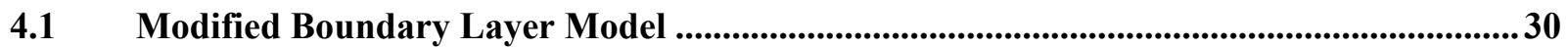

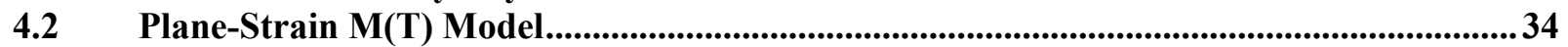

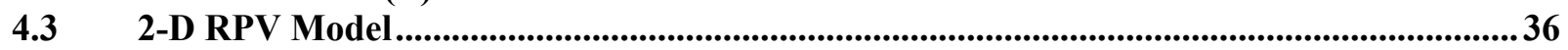

4.4 Weibull Stress Approximation Using 2-D Models ...................................................................... 38

$5 \quad$ Summary and Conclusions ......................................................................................................................... 43

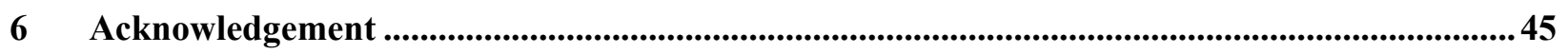

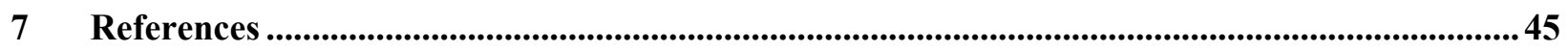




\section{LIST OF FIGURES}

Figure 1 (a) An embedded flaw in the wall of an RPV; (b) Weibull stress evolution at point D over a thermal-pressure transient; (c) Weibull stress evolution at point $\mathrm{C}$ over a thermal-pressure transient; and (d) $\left(\sigma_{\mathrm{w}}-\sigma_{\mathrm{min}}\right) /\left(\sigma_{\mathrm{u}}-\sigma_{\mathrm{min}}\right)$ over the crack front at $t=50 \mathrm{~min}$ of the thermal-pressure transient.

Figure 2 Geometric configuration of: (a) a quarter symmetric model of a reactor pressure vessel; and (b) an embedded flaw in the wall of the RPV.

Figure 3 Room temperature, uniaxial, true stress-logarithmic strain relationships for: (a) the vessel wall (ferritic) steel characteristic of ASTM A533B; and (b) the austenitic stainless steel for the cladding.

Figure 4 The PTS transient condition: (a) coolant temperature; and (b) internal pressure. Linear interpolations, indicated by the solid line, smooth these loading histories for input to the fracture analysis.

Figure 5 Temperature variations over the wall thickness at time intervals of 5 minutes computed by the 1-D axisymmetric analysis in FAVOR.

Figure 6 The quarter-symmetric finite element model using 20-node brick elements for an RPV with an axially embedded elliptical flaw: (a) global model; (b) close-up view for the embedded crack with 30 elements along the semi-elliptical crack front; (c) each crack-front position has 31 rings of elements; and (d) the initial root diameter of the crack front equals $6 \mu \mathrm{m}$.

Figure 7 (a) Variation of the linear-elastic stress intensity factor; (b) variation of the linearelastic $T$-stress; and (c) variation of the non-dimensional $\beta$ ratio. 26

Figure 8 (a) Evolution of the Weibull stress values computed over the entire perimeter of the crack for the transient history; and the variation of local (pointwise) Weibull stresses at $t=0,10$ min and 18 min over the crack front for (b) $m=10$; (c) $m=15$; and (d) $m=20$.

Figure 9 (a) Configuration of the modified boundary layer (MBL) model; and crack-front stresses at $\boldsymbol{P}_{\text {int }}=\boldsymbol{P}_{0}$ and $\boldsymbol{P}_{\text {int }}=2 \boldsymbol{P}_{0}$ for (b) point A; (c) point B; and (d) point C; along the 3-D curved crack front.

Figure 10 (a) Configuration of the M(T) model; (b) FE mesh for the M(T) model; comparison of the crack-front stress, $\sigma_{y y}$, from the plane-strain $M(T)$ model and from the embedded crack in the 3D RPV under: (c) internal pressure only; and (d) combined thermal-pressure transient. 35

Figure 11 Evolution of the $T$-stress biaxiality ratio, $\beta$, over the PTS transient history at: (a) points B, C, D, and E with low constraints; (b) point A with high constraints; and (c) locations of the different crack-front points.

Figure 12 (a) Finite element meshes for a plane-strain, RPV model (actually a 3-D model with one-layer of elements and plane-strain constraints); and comparison of the crack-front stress, $\sigma_{y y}$, 
computed from the plane-strain RPV model and from the 3-D RPV model: (b) at the crack-front point $B$; and (c) at the crack-front point $C$

Figure 13 Evolution of the elastic-plastic $K_{J}$-values over the transient history computed from the plane-strain RPV model and from the 3-D RPV model for: (a) point B; and (b) point C; along the curved crack front.

Figure 14 Evolution of the Weibull stress over the transient history computed from the planestrain RPV model and from the 3-D RPV model for: (a) point B; (b) point C; (c) point D; and (d)

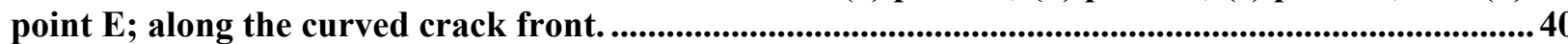

Figure 15 Evolution of the Weibull stress over the transient history computed from the planestrain 2-D RPV model and from the 3-D RPV model for point A along the curved crack front....... 41 


\section{NOMENCLATURE}

$B=$ thickness of a fracture specimen (crack front length)

$\tilde{C}=$ constant defining the relationship between the Weibull stress value and the fracture parameter, $K_{J}$, determined for small-scale yielding conditions

$E=$ elastic modulus

$J=$ elastic-plastic energy release rate

$J_{\text {Jac }}=$ Jacobian between the global and local coordinate systems

$K_{I}=$ mode I stress intensity factor

$K_{J}=$ stress intensity factor computed from the domain $J$-integral $\left(=\sqrt{E J /\left(1-v^{2}\right)}\right.$ for plane-strain condition)

$K_{\min }=$ threshold fracture toughness below which cleavage fracture does not occur

$K_{0}=$ fracture toughness scale parameter in ASTM E-1921

$L=$ crack-front length

$M=$ non-dimensional loading parameter $\left(=b \sigma_{0} / J\right)$

$P_{f}=$ cumulative probability of failure

$P_{\text {int }}=$ internal pressure acting on the RPV

$P_{0}=$ initial internal pressure acting on the RPV at $t=0$

$R=$ radius of the modified boundary layer model

$R_{0}=$ initial root radius of the crack-front finite element mesh

$T$ = elastic $T$-stress

$V_{0}=$ reference volume in the Weibull stress calculation

$V_{f}=$ volume of the fracture process zone

$W=$ width of the fracture specimen

$a=$ crack depth

$c=$ half crack length

$d=$ distance from the base metal-cladding interface to the nearest crack front

$f_{i j}=$ function defining the angular variation of the singular crack-front stresses

$g=$ non-dimensional constraint correction function for Weibull stress computations

$m=$ Weibull stress modulus

$n=$ number of segments along the crack front

$r_{\text {int }}=$ internal radius of the RPV

$r_{\text {ext }}=$ external radius of the RPV

$t=$ time over a transient

$u=$ displacement in the $x$-direction

$v=$ displacement in the $y$-direction

$(x, y, z)=$ Cartesian coordinate system with the origin at the crack tip

$\Delta_{\text {CMOD }}=$ crack mouth opening displacement 
$\alpha=$ constant defining the relationship between crack-tip opening displacement and $J$ integral

$\beta=$ non-dimensional biaxiality ratio $\left(=T \sqrt{\pi a} / K_{I}\right)$

$\theta=$ counter-clockwise angular measure from the horizontal axis originating at the crack tip

$\lambda=$ stress cutting parameter in the Weibull stress model ( $=2$ in this study)

$\sigma_{H}=$ hydrostatic stress $\left[=\left(\sigma_{11}+\sigma_{22}+\sigma_{33}\right) / 3\right]$

$\sigma_{\text {eff }}=$ effective stress in the Weibull stress model ( $=\sigma_{1}$ in this study)

$\sigma_{r r}=$ radius stress in the wall of an RPV

$\sigma_{\theta \theta}=$ hoop stress in the wall of an RPV

$\sigma_{u}=$ Weibull stress scaling parameter (= microscale material toughness)

$\sigma_{w}=$ Weibull stress

$\sigma_{w-\min }=$ threshold Weibull stress below which cleavage fracture does not occur

$\sigma_{0}=$ uniaxial, tensile yield stress

$\sigma_{1}, \sigma_{2}, \sigma_{3}=$ principle stresses with $\sigma_{3} \leq \sigma_{2} \leq \sigma_{1}$

$v=$ Poisson's ratio

$\eta=$ dimensionless constant

$\left(\eta_{1}, \eta_{2}, \eta_{3}\right)=$ local (parametric) element coordinate system 


\title{
Cleavage Fracture Modeling of Pressure Vessels under Transient Thermo-Mechanical Loading
}

\author{
Xudong Qian \\ Department of Civil Engineering, National University of Singapore, Singapore 117576 \\ Robert H. Dodds Jr. \\ Department of Civil and Environmental Engineering, \\ University of Illinois at Urbana Champaign, IL 61801 \\ Shengjun Yin and Richard Bass \\ Oak Ridge National Laboratory, PO Box 2008 MS6085, Oak Ridge, TN 37831
}

\begin{abstract}
The next generation of fracture assessment procedures for nuclear reactor pressure vessels (RPVs) will combine nonlinear analyses of crack-front response with stochastic treatments of crack size, shape, orientation, location, material properties and thermal-pressure transients. The projected computational demands needed to support stochastic approaches with detailed 3-D, nonlinear stress analyses of vessels containing defects appear well beyond current and near-term capabilities. In the interim, 2-D models become appealing to approximate certain classes of critical flaws in RPVs, and have computational demands within reach for stochastic frameworks. The present work focuses on the capability of 2-D models to provide values for the Weibull stress fracture parameter with accuracy comparable to those from very detailed 3-D models. Weibull stress approaches provide one route to connect nonlinear vessel response with fracture toughness values measured using small laboratory specimens. The embedded axial flaw located in the RPV wall near the cladding-vessel interface emerges from current linear-elastic, stochastic investigations as a critical contributor to the conditional probability of initiation. Three different types of 2-D models reflecting this configuration are subjected to a thermal-pressure transient characteristic of a critical pressurized thermal shock event. The plane-strain, 2-D models include: the modified boundary layer (MBL) model, the middle tension (M(T)) model, and the 2-D RPV model. The 2-D MBL model provides a high quality estimate for the Weibull stress but only in crack-front regions with a positive $T$-stress. For crack-front locations with low constraint ( $T$-stress $<0$ ), the $\mathrm{M}(\mathrm{T})$ specimen provides very accurate Weibull stress values but only for pressure load acting alone on the RPV. For RPVs under a combined thermal-pressure transient, Weibull stresses computed from the 2-D RPV model demonstrate close agreement with those computed from the corresponding crack-front locations in the 3D RPV model having large negative T-stresses. Applications of this family of 2-D models provide Weibull stress values in excellent agreement with very detailed 3-D models while retaining practical levels of computational effort.
\end{abstract}




\section{Introduction}

Reactor pressure vessels (RPVs) consist of thick-walled, cylindrical steel structures with hemispherical heads that enclose the core in a nuclear power plant. RPVs experience a wide range of complex, thermalmechanical loading cycles during their service life, now extending past 30 years for increasing numbers of plants. Destructive evaluation of newly manufactured RPVs for research purposes [1-3] have revealed a distribution of crack-like flaws, with variable size, shape, orientation and location, which can be expected for those RPVs in service. The RPVs must function safely under both normal operating conditions as well as severe conditions caused by accidents. Pressurized thermal shock (PTS) represents one such accident scenario that imposes severe cooling concurrent with or followed by significant pressure changes in the vessel. Combined with a reduction of fracture toughness from years of radiation embrittlement, the thermal-pressure transients during a PTS event can define critical fracture conditions for vessel integrity. Increasingly, assessments of vessel integrity employ conventional linear-elastic fracture mechanics (LEFM) combined with a stochastic treatment of crack size, shape, orientation, location, material properties, and details of the thermal-pressure transient (e.g., as implemented in the ORNL-FAVOR code [4-5]). Brittle, cleavage fracture represents the failure mechanism of major concern in these assessments. However, the critical combination of material flow-toughness properties, crack sizelocation-orientation and loading likely leads to inelastic deformations sufficient to impact significantly the assumptions inherent in LEFM.

The probabilistic Weibull stress $\left(\sigma_{w}\right.$ ) framework for cleavage fracture, originally proposed by the Beremin group [6,7], considers material toughness and loading "local” to the immediate crack front region and thus applies when $K_{I}$ or the $J$-integral no longer describe the crack front displacement-strainstress fields under increasing plastic deformation. The fracture process zone represents small, but finite, volumes of material which fully embody a population of microscale flaws, whose size and density constitute material properties. The statistical distribution of microcracks governs the cumulative probability of fracture, which can be modeled using a Griffith-type fracture criterion imposed on the critical microcrack size [8]. The Weibull stress framework has become a generally recognized approach to quantify the probabilistic driving force for cleavage fracture [9-12], to characterize material toughness properties in a probabilistic [13, 14], and to develop and apply constraint corrections [15-17] which accommodate differences in crack-front conditions between small laboratory test specimens and RPVs. Recent work by Petti and Dodds [18] suggests that coupling of the Weibull stress approach and the macroscopic Master Curve concept (as outlined in ASTM E-1921 [19]) can dramatically reduce the laboratory testing of fracture specimens required for calibration of material parameters in the Weibull 
stress model for specific ferritic steel. The outcome is a new modeling process that approaches the simplicity of an "engineering” assessment for cleavage fracture integrity of an RPV.

Computation of Weibull stress values requires elastic-plastic, large deformation analyses of 3-D RPV models using very detailed crack-front finite element meshes. Both the mesh preparation effort and the computation time for analyses immediately grow to impractical levels for detailed assessments of RPVs over a range of flaw geometries, locations, orientations, shapes and configurations subjected to a spectrum of thermal-mechanical transients. Moreover, the PTS triggered thermal-pressure transients impose both spatial and temporal variations of temperatures over the wall thickness. Consequently, both the local crack-front driving force $\left(\sigma_{w}\right)$ and the material properties, characterized by the Weibull parameters, vary along the curved front of a 3-D flaw. This contrasts to much simpler conditions forming the basis of E-1921: high constraint geometries, through-thickness cracks with relatively straight fronts, and constant temperature within specimens leading to invariant toughness along the crack fronts. Engineering applications of the Weibull stress framework, for example, to elliptical flaws embedded in the wall of an RPV near the cladding require both accurate computations of the Weibull stress values and detailed calibrations of the temperature dependent/independent Weibull material parameters along the 3D crack front over the entire transient history.

These complexities and the impractical computational expense to conduct nonlinear 3-D, stochastic simulations of the type now performed in FAVOR using LEFM drive the consideration and development of approximate 2-D computational models to estimate Weibull stress values over the PTS transient. The approximation of certain types of 3-D configurations with simpler 2-D models relies on the similitude of asymptotic stress fields near the tip of a 2-D crack model with those of a 3-D crack model, both of which are subjected to the same linear-elastic $K_{I}$ and $T$-stress at the crack-front location of interest. The modified boundary layer model (MBL) provides the computational framework for the $K_{I}$ and $T$-stress approach. In other cases, a 2-D finite-body configuration becomes necessary. Cravero and Ruggieri [20] and Silva et al. [21] employ plane-strain, single-edge notched tension (SE(T)) models to represent the crack-front stress fields near an axial, surface-breaking crack in the wall of a pipeline. For embedded, axially-oriented cracks in the wall of an RPV, the MBL or a plane-strain, M(T) model, would seem appropriate 2-D approximate models.

The current study examines different types of 2-D (plane-strain) models to approximate the stress fields along the front of an embedded flaw in the wall of an RPV subjected to a complex thermal-mechanical transient characteristic of a PTS event. Elastic-plastic, large deformation analyses of the full 3-D RPV model with an axially embedded crack employ very detailed finite element meshes built with 20-node brick elements. The Weibull stress values computed from the detailed 3-D RPV model provide the 
reference cases to benchmark various, simplified 2-D models. The current investigation considers three 2D models, namely the modified boundary layer (MBL) model, the $\mathrm{M}(\mathrm{T})$ model and the 2-D cylinder (RPV) model. The numerical results and discussion focus on the capability of the 2-D models to provide Weibull stress values having very close agreement with Weibull stress values obtained from the 3-D analyses. Highly accurate Weibull stress values form the prerequisite for realistic estimates of the cumulative probability of fracture. Comparisons are made separately for pressure load acting alone and for combined thermal-pressure loadings of the PTS event to understand the differences in behavior of 2-D vs. 3-D models.

The report begins with a description of the Weibull stress framework, and introduces the key parameters and computational concepts. The following section discusses the 3-D modeling and numerical procedures to compute global and point-wise Weibull stress values for an elliptical axial crack embedded in the wall of an RPV. The subsequent section examines the applicability of the three 2-D simplified models to estimate Weibull stress values. The final section summarizes the main findings in the current study.

\section{Weibull Stress Framework}

\subsection{Weibull Stress Model}

The large scatter observed in experimentally measured fracture toughness (e.g., $\left.K_{J}\right)$ values for ferritic steels over the ductile-to brittle transition (DBT) region has driven the development of probabilistic treatments of the data. The widely adopted "weakest link model” assumes that fracture of a material volume depends on a single initiator [22-24], or equivalently a single microcrack. Weakest link concepts form the technical basis for the first testing standard developed specifically to address the unique statistical issues with ferritic steels at temperatures over the DBT region (ASTM E1921 [19]). The macroscopic fracture model, as outlined in ASTM E-1921, assumes that the temperature, the local $J$ values, and the local stress fields remain essentially uniform along the entire crack front and that smallscale yielding (SSY) conditions prevail at the cleavage fracture event. SSY conditions insure the unique correspondence across specimens (specified by ASTM E-1921) between the crack-front $J$-value and the local crack-front strains-stresses at fracture. The corresponding microscopic fracture model employs directly the strains-stresses at each crack-front location. SSY conditions then become unnecessary to establish the link between the local crack-front stress fields and the scalar measure $(J)$ of the loading. The Beremin group [6] introduced the most widely used microscopic model. In this model, a local fracture parameter, the so-called Weibull stress $\left(\sigma_{w}\right)$, defines an integrated, scalar measure of the crack-front conditions driving cleavage fracture at increasing levels of external loading. Numerical analyses connect 
values of $\sigma_{w}$ with external loading of the specimen-structure and may include complexities from variable crack-front geometry, mismatched material properties, large-scale yielding, thermal-mechanical loading, etc.

By correlating an assumed, inverse-power density distribution of critical microcrack sizes with the local stress through a simple fracture mechanics model, the Beremin group [6] derived the cumulative failure probability for a two-parameter Weibull stress model,

$$
P_{f}\left(\sigma_{w}\right)=1-\exp \left[-\left(\frac{\sigma_{w}}{\sigma_{u}}\right)^{m}\right]
$$

where $m$ is the Weibull modulus, the value of which depends on the statistical distribution of microcrack sizes in the material, and the scalar Weibull stress, $\sigma_{w}$, follows,

$$
\sigma_{w}=\left[\frac{1}{V_{0}} \int_{V_{f}} \sigma_{e f f}^{m} d V_{f}\right]^{1 / m}
$$

The scale parameter, $\sigma_{u}$, denotes the Weibull stress value at a cumulative fracture probability of 0.632 , or $P_{f}\left(\sigma_{w}=\sigma_{u}\right)=0.632$. $V_{0}$ defines the (normalizing) reference volume and $V_{f}$ represents the fracture process zone. The practical approach to compute the Weibull stress employs numerical integration of an "effective" stress measure, $\sigma_{\text {eff }}$, over the fracture process zone at each loading level imposed on a finite element model of the cracked configuration. The effective stress denotes a local "driving force" acting on statistically independent (small) volumes of material surrounding the crack front. The $\sigma_{\text {eff }}$ selection depends on the local fracture criterion, and most often takes the value of the maximum principal stress, $\sigma_{1}$, or the hydrostatic stress, $\sigma_{H}=\left(\sigma_{1}+\sigma_{2}+\sigma_{3}\right) / 3$. The current study adopts $\sigma_{1}$ as the effective stress.

The fracture process zone contains a region of plastically deformed, crack-front material over which the effective stress exceeds a characteristic value, $\lambda \sigma_{0}$, with a typical value of $\lambda=2$ (here $\sigma_{0}$ denotes the uniaxial, tensile yield stress). The normalizing volume, $V_{0}$, cancels in applications of the Weibull stress model to compare crack-front conditions for the same material (e.g. between small lab specimens and structures) and takes a value of unity here for simplicity. Numerical evaluation of the Weibull stress follows, 


$$
\sigma_{w}=\left[\frac{1}{V_{0}} \sum_{n_{e}} \int_{-1}^{1} \int_{-1}^{1} \int_{-1}^{1} \sigma_{e f f}^{m}\left|J_{J a c}\right| d \eta_{1} d \eta_{2} d \eta_{3}\right]^{1 / m}
$$

where $n_{e}$ denotes the number of elements inside the fracture process zone, and $\left|J_{J a c}\right|$ defines the determinant of Jacobian between the global coordinate system and the element local (parameters) coordinate system.

The computed Weibull stress values reflect both the magnitude of the local crack-front stresses and the extent of the fracture process zone ahead of the crack tip. The Weibull stress values, therefore, incorporate the effects of local stress changes caused by the varying levels of constraint on local plastic flow in the immediate crack front region. Low constraint conditions reduce the stress triaxiality within the plastic zone, and therefore decrease the effective stress value within the fracture process zone. Consequently, a fracture specimen with low constraint requires a potentially much larger driving force, measured in terms of $K_{J}$, than is needed for a high-constraint fracture specimen, to achieve the same $\sigma_{w}$ and consequently the same probability of fracture (see Eq. 1). The probability of fracture expressed in terms of the macroscopic crack driving force, e.g., $K_{J}$, applies strictly to (typically high) crack-front constraint conditions from which the probability function derives in E-1921. Extrapolation of such a probability function to a crack-front with low constraint conditions leads to incorrect estimates of failure probabilities. In contrast, the probability of fracture, expressed in the local driving force, $\sigma_{w}$, predicts the same probability for equal $\sigma_{w}$-values irrespective of the crack-front constraint levels.

Estimates of the cumulative probability for cleavage fracture must reflect the actual, often complex, loading history imposed on crack front material. The Weibull stress framework assumes that a reduction in the crack-front stress levels during the loading history does not "heal" the damage to material caused by the previous higher levels of stress. Consequently, the decrease in Weibull stress values computed from Eq. 3 due to local unloading, and thus a reduced level of crack front stresses, does not decrease the cumulative probability of fracture. When local unloading does occur, the numerical procedures simply retain the largest, previous value computed for the Weibull stress. The Weibull stress thus becomes a stationary or monotonically increasing function of the imposed loading history.

\subsection{Calibration of Weibull Stress Parameters}

The original two-parameter Weibull stress model in Eq. 1 represents a microscale model to compute the cumulative probability of fracture with the Weibull stress parameters $m$ and $\sigma_{u}$ characterizing microscopic material properties. The calibration of $m$ and $\sigma_{u}$ through measurements of microscale material quantities demands formidable and expensive experimental efforts. On a macroscopic level, 
however, the cumulative probability of fracture computed from the Weibull stress framework should predict the cumulative probability expression adopted in ASTM E-1921 applicable for high constraint conditions,

$$
P_{f}\left(K_{J}\right)=1-\exp \left[-\left(\frac{K_{J}-K_{\min }}{K_{0}-K_{\min }}\right)^{4}\right]
$$

where $K_{0}$ defines a temperature dependent, toughness scale parameter and $K_{\min }$ denotes the threshold fracture toughness value below which the probability of cleavage fracture becomes zero. ASTM E-1921 suggests a constant value of $K_{\text {min }}=20 \mathrm{MPa} \sqrt{\mathrm{m}}$ for ferritic steels independent of the geometry of the specimen/structure and temperature. Through a coupling the macroscopic three-parameter expression in Eq. 4, Petti and Dodds [18] propose a Weibull stress-based, three-parameter cumulative probability of fracture model, which requires a minimum threshold Weibull stress to obtain non-zero probabilities of fracture, as given in Eq. 5,

$$
P_{f}\left(\sigma_{w}\right)=1-\exp \left[-\left(\frac{\sigma_{w}^{m / 4}-\sigma_{w-\min }^{m / 4}}{\sigma_{u}^{m / 4}-\sigma_{w-\min }^{m / 4}}\right)^{4}\right]
$$

The minimum (threshold) Weibull stress, $\sigma_{w-\min }\left[\sigma_{w-\min }=\sigma_{w}\left(K_{J}=K_{\min }\right)\right]$, depends on the crack-front length, material flow properties, temperature and Weibull parameters $\left(m, V_{0}\right)$ [18]. For a fixed exponent $(m)$, Petti and Dodds [25] demonstrate that the Weibull scaling parameter, $\sigma_{u}$, increases with increasing temperature, which reflects the increasing microscale toughness of ferritic steels caused by local events that include plastic shielding of microcracks, microcrack blunting and microcrack arrest.

The comparison of Eqs. 4 and 5 implies the following relationship between the Weibull stress and the macroscopic crack driving force, $K_{J}$,

$$
\sigma_{w}^{m}=\tilde{C} B K_{J}^{4} g(M, m)
$$

where $M=b \sigma_{0} / J$ is a monotonically decreasing measure of loading, and $b$ denotes the remaining ligament length of the fracture specimen. The function $g(M, m) \leq 1$ quantifies the constraint correction with respect to a reference small-scale yielding condition without constraint loss ( $T$-stress $=0$ ). For high constraint specimens without any constraint loss, $g(M, m)=1$. The constant $\tilde{C}$ derives from the 
relationship between the Weibull stress and the macroscopic $K_{J}$-value computed using the reference SSY model (with $g(M, m)=1$ ). The parameter $B$ denotes the crack-front length.

The correlation between the Weibull stress and crack-front $K_{J}$-values in Eq. 6 provides the engineering basis to calibrate the Weibull stress parameters, $m, \sigma_{u}$ and $\sigma_{w-\min }$ (or $K_{\min }$ ). The engineering procedure to calibrate the Weibull stress parameters, as originally proposed by Gao et al. [11], employs measured fracture toughness values obtained for high constraint (SSY) and low constraint [large scale yielding (LSY)] configurations. The calibrated set of Weibull parameters, $m$ and $\sigma_{w-\min }$, scales the Weibull stress values computed from the high-constraint specimens and those from the low constraint specimens to the same $P_{f}-K_{J}$ curve corresponding to a standard, small-scale yielding condition. The Weibull scale parameter, $\sigma_{u}$, equals the Weibull stress value when $K_{0}$ replaces $K_{J}$ in Eq. 6. Petti and Dodds [25] utilize the Master Curve characterization of the macroscopic toughness ( $\left.K_{0}\right)$ vs. temperature relation [19] obtained by as few as six fracture specimens at one temperature to calibrate the Weibull scale parameter, $\sigma_{u}$, as a function of temperature over the DBT region. They demonstrate through measured properties of two pressure vessel steels (A533B and A508) that the scale parameter, $\sigma_{u}$, increases significantly with temperature for a fixed Weibull modulus, $m$, in a three-parameter Weibull stress model. Wasiluk et al. [26] present a calibration study of the three parameter Weibull stress model for a 22Ni-MoCr37 pressure vessel steel, utilizing the large-scale datasets from a European Union research project - "Fracture toughness of steel in the ductile to brittle transition regime" [27, 28]. Wasiluk et al. [26] demonstrate an effective, temperature invariance of the Weibull modulus, $m$, and a temperature dependence of the Weibull scale parameter, $\sigma_{u}$, through calibrations at two extreme temperatures of the ductile-to-brittle transition regime for this common RPV steel. The calibration outcome for this steel also suggests that $K_{\min }$ increases gradually with temperature, in contrast to the simpler assumption of a fixed value of $K_{\min }=20 \mathrm{MPa} \sqrt{\mathrm{m}}$ adopted in E-1921.

\subsection{Application to 3-D Vessel Flaws}

In typical research applications of the Weibull stress framework conducted to date, the laboratory specimens have relatively straight, through-thickness cracks with constant temperature at all locations along the front. Consequently, the Weibull stress parameters, $\sigma_{u}$ and $\sigma_{w-m i n}$, and material flow properties remain constant at all crack-front locations. Local $K_{J}$-values along the crack front remain relatively uniform except for much lower values over the thin boundary layer transition at the outside surface of the specimens. The 3-D finite element analyses that accompany these tests readily yield a thickness average $K_{J}$-value and the corresponding (single) $\sigma_{w}$-value at each level of applied loading. The $K_{J}$-value

corresponds to the value found using the measured $P-\Delta_{\text {СмоD }}$ curve and an $\eta$-value for the specimen configuration, or through direct, 3-D finite element analysis of the specific specimens. Experimental and 
computational studies using the measured $K_{J c}$-value and corresponding $\sigma_{w}$-value are thus greatly simplified by the constant $\sigma_{u}$ and $\sigma_{w-\min }$ values, and flow properties along the crack front.

The conditions for an embedded crack in the wall of an RPV, as exemplified in Fig. 1(a), under timedependent, thermal-mechanical loadings become far more complex. Details of the geometry, loading conditions, and computational procedures of this RPV model follow the description presented in the next section. Figures 1(b) and 1(c) illustrate significant differences in the evolution of Weibull stresses at two different locations, denoted $\mathrm{C}$ and $\mathrm{D}$, along the 3-D curved crack front for a realistic thermal-pressure transient. The thermal-pressure transient creates variations in the temperature and thus in $\sigma_{u}$ and $\sigma_{w-\min }$ values along the crack front. Similarly, $\sigma_{u}, \sigma_{w-\min }$ and flow properties likely also vary along the crack front from differing levels of material damage caused by accumulated radiation exposure over years of service life. This potentially strong variation in local fracture and flow properties along the crack front, coupled with strong variations in crack-front loading leads to a very complex situation to apply the Weibull stress framework (or any other framework). In essence, each segment of material along the front of an embedded crack is effectively a small "component” with its own loading history and time varying flow and toughness properties $\left(\sigma-\varepsilon ; K_{0}, \sigma_{u}\right.$ and $\left.\sigma_{w-m i n}\right)$, e.g., for points C and D in Fig. 1(a). The assumption (now apparently validated) of a temperature invariant exponent, $m$, does provide some much needed simplification, since the Weibull stress values exhibit strong dependence on the $m$-value as shown in Figs. 1(b) and 1(c).

The potentially significant spatial and temporal changes both in the material properties and in the local crack driving force, caused by the thermal transient, require detailed mathematical descriptions for an accurate estimation of the cumulative probability of failure. The thermal loading changes the mechanical properties of the material along the crack front and impacts the Weibull scaling parameter, $\sigma_{u}$, with a smaller impact on the threshold Weibull stress, $\sigma_{w-\min }$. Meanwhile, the local Weibull stress changes significantly along the crack front, as demonstrated by the comparison of Figs. 1(b) and 1(c). To include the variation of material properties along the crack front, a refined procedure for PTS assessment divides material along the entire crack front into $n$ small subregions each with length $L_{(i)}(i=1,2, \ldots n)$. Each subregion thus experiences a spatially constant temperature and a spatially constant loading $\sigma_{w_{(i)}}$. The total cumulative failure probability, proposed in a previous study [29], follows as, 


$$
P_{f}^{\text {Total }}\left(t_{k}\right)=1-\exp \left[-\sum_{i=1}^{n}\left(\frac{\sigma_{w_{(i)}^{m}}^{m / 4}-\sigma_{w-m i n}^{m / 4}}{\sigma_{u_{(i)}}^{m / 4}-\sigma_{w-m i n}^{m / 4}}\right)^{4}\right]
$$

where $\sigma_{u_{(i)}}$ and $\sigma_{w-m_{n}(i)}$ denote the Weibull scaling parameter and the threshold Weibull stress parameter of the subregion $i$. Equation 7 defines the total, cumulative failure probability of the embedded flaw in the RPV at a specific time $t_{k}$ of the transient. Equation 7 follows the weakest link model which assumes that a single initiator within a segment triggers cleavage fracture along the entire crack front.

Figure 1(d) shows the ratio of $\left(\sigma_{w}-\sigma_{w-\min }\right) /\left(\sigma_{u}-\sigma_{w-\min }\right)$ for different segments along the crack front at $t=50 \mathrm{~min}$ into the thermal-pressure transient, when the Weibull stress along the crack front maintains the maximum value achieved at $t=18 \mathrm{~min}$ and the material toughness, $\sigma_{u}$, takes on the lowest values. Figure 1(d) indicates that only a small fraction of the entire crack front contributes to the total probability of fracture since cleavage fracture does not occur when $\sigma_{w} / \sigma_{w-\min } \leq 1.0$. Consequently, only the stressstrain-displacement results from a small fraction of the curved crack front in the very detailed 3-D RPV model contributes to the local fracture driving force that is sufficiently large to cause a nonzero probability of fracture. This motivates the development of computationally more efficient 2-D procedures to approximate the Weibull stress values in the critical front locations along the 3-D embedded crack where $\sigma_{w} / \sigma_{w-\min }>1.0$.

Similitude in the stress-strain fields near two different crack tips under the same crack-front loading (e.g., $K_{I}$ and $T$ stresses) provides the theoretical basis for simplified 2-D finite element models to approximate the Weibull stress values along the 3-D crack front. A “correct” 2-D model predicts the stress field near the corresponding location along the 3-D crack front to estimate accurately the Weibull stress values. However, low constraint near the crack front and combined thermal-pressure transients over the wall thickness of an RPV introduce additional challenges for 2-D modeling frameworks when large-scale yielding develops. The criterion adopted here to identify an appropriate 2-D model relies on the matching of Weibull stress values computed from the detailed 3-D RPV analysis discussed in the following section. 
(a)
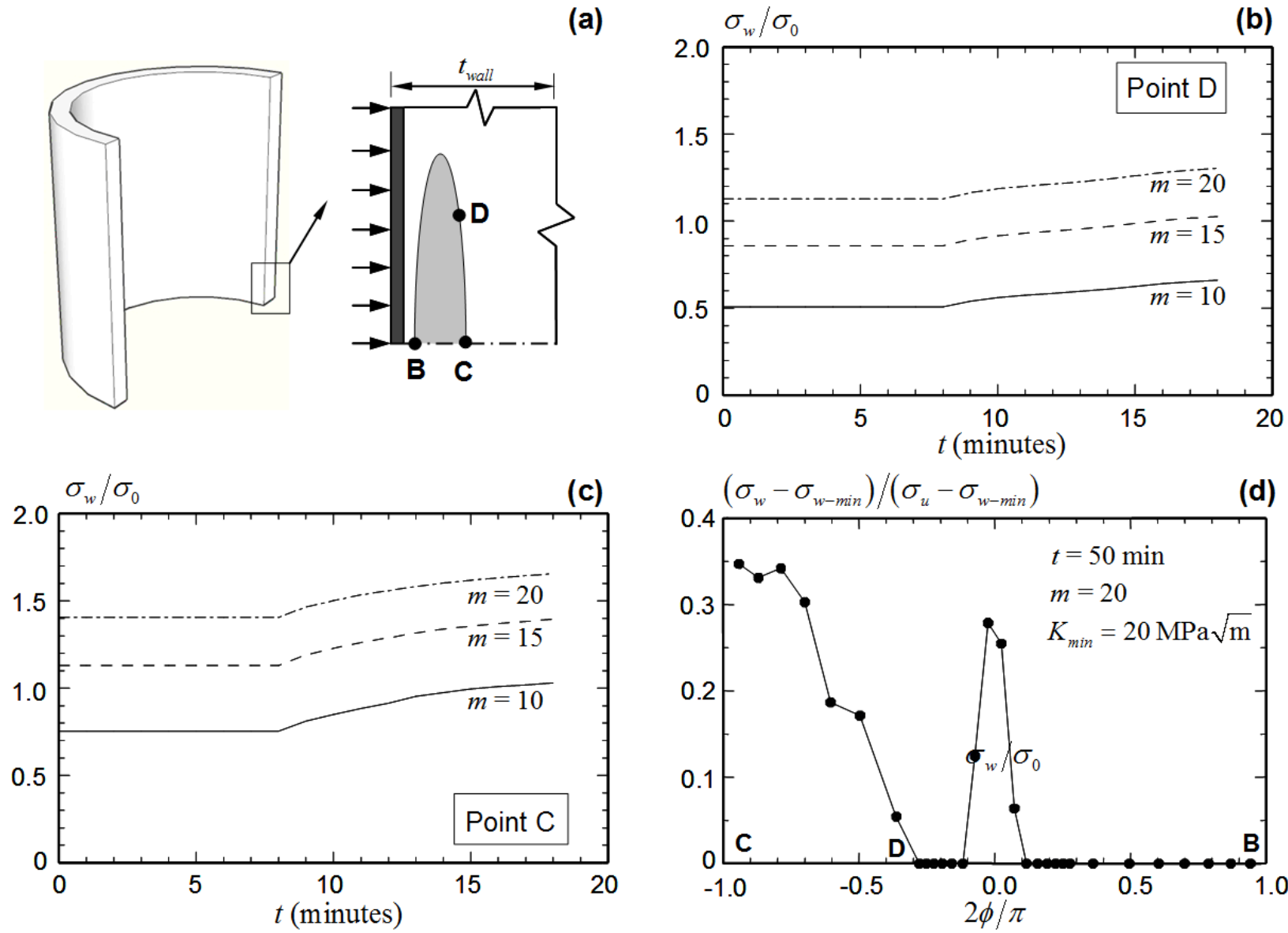

Figure 1 (a) An embedded flaw in the wall of an RPV; (b) Weibull stress evolution at point D over a thermal-pressure transient; (c) Weibull stress evolution at point $C$ over a thermalpressure transient; and (d) $\left(\sigma_{\mathrm{w}}-\sigma_{\mathrm{min}}\right) /\left(\sigma_{\mathrm{u}}-\sigma_{\mathrm{min}}\right)$ over the crack front at $t=50 \mathrm{~min}$ of the thermal-pressure transient.

\section{3-D RPV Models and Results}

This section describes the geometry, material properties, thermal-pressure transients, and finite element models for an RPV with an axially embedded flaw. Discussions of the computational results focus on the evolution of constraint conditions along the crack front and the Weibull stress values that drive assessment of the cumulative probability of cleavage fracture.

\subsection{Geometry}

Figure 2 shows the schematic configuration of a quarter-symmetric RPV model investigated in the current study. The vessel has this geometry: inner radius of the RPV $r_{\text {int }}=1994 \mathrm{~mm}$, wall thickness of $t_{\text {wall }}=204$ $\mathrm{mm}$, additional cladding thickness of $4 \mathrm{~mm}$, and height of the cylindrical part of the RPV equals $7 \mathrm{~m}$. The 
crack surface of the embedded, planar flaw aligns with the axial direction of the RPV. The elliptical axial flaw has an aspect ratio of $a / c=0.17$ with crack depth $2 a=17 \mathrm{~mm}$ in the wall thickness direction. The inside edge of the axial crack lies a distance $d=2 \mathrm{~mm}$ from the cladding (see Fig. 2) or $6 \mathrm{~mm}$ from the inner surface of the RPV. A previous investigation [30] using stochastic simulations demonstrates that this flaw size and location contributes significantly to the conditional probability of initiation (CPI). The inner surface of the base RPV has a thin, $4 \mathrm{~mm}$ layer of austenitic stainless steel to protect the base material (made of the ferritic steel) from corrosion.

\section{Quarter-symmetric Model}

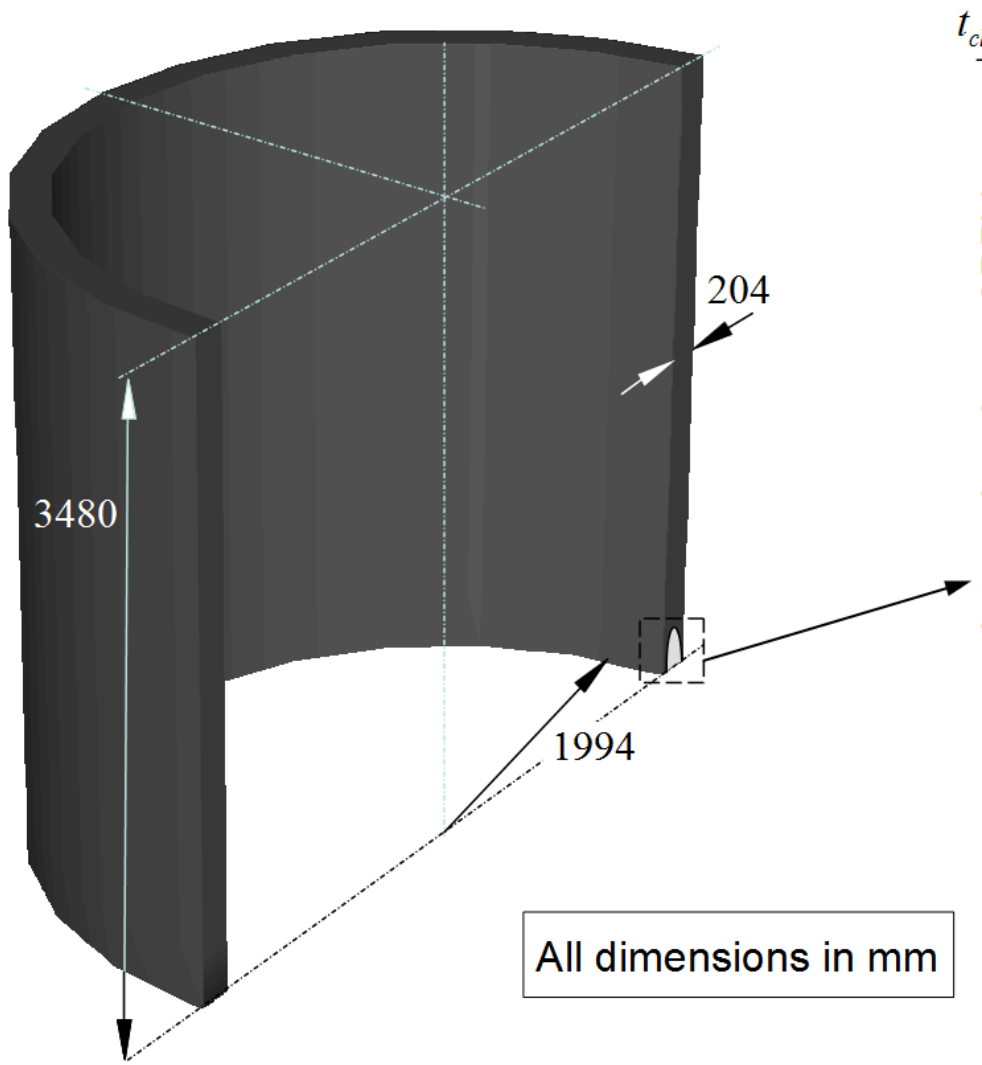

(b)

(a)

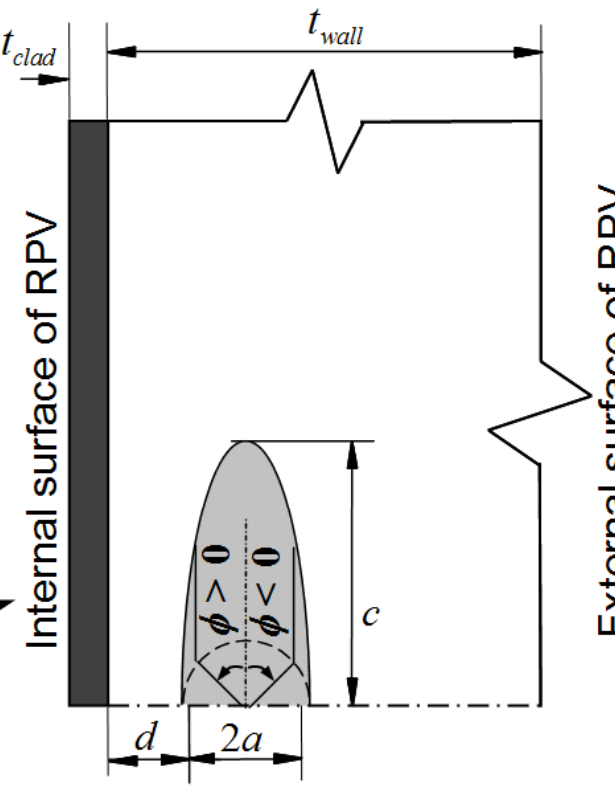

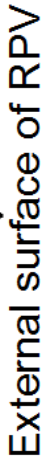

Figure 2 Geometric configuration of: (a) a quarter symmetric model of a reactor pressure vessel; and (b) an embedded flaw in the wall of the RPV.

\subsection{Material Properties}

The properties of the base material for the RPV are representative of an ASTM-A533B ferritic steel, with typical values of room temperature yield strength $\sigma_{y}=500 \mathrm{MPa}$, Young's modulus $E=193 \mathrm{GPa}$, and coefficient of thermal expansion (CTE) of $14 \times 10^{-6} 1 /{ }^{\circ} \mathrm{C}$. The austenitic stainless steel cladding has a room temperature yield strength $\sigma_{y}=163 \mathrm{MPa}$, Young's modulus $E=157 \mathrm{GPa}$, and a larger CTE value of $17 \times 10^{-6} 1 /{ }^{\circ} \mathrm{C}$. The severe mismatch in yield strength and the smaller mismatch in CTE between the 
base material and the cladding steel create differential plastic deformation and thermal expansion near the base-cladding interface under combined thermal-mechanical loadings. Figure 3 shows the uniaxial true stress - logarithmic strain relationship for the ferritic base steel and the austenitic cladding steel. The maximum value of 2.0 for the logarithmic strain in the base steel improves convergence features of the solutions near the crack front which experiences large plastic deformations. The present analyses adopt temperature invariant material properties for the base and cladding steels. Temperature dependent material properties would not alter the conclusions on the applicability of various simplified models to compute the Weibull stress values.

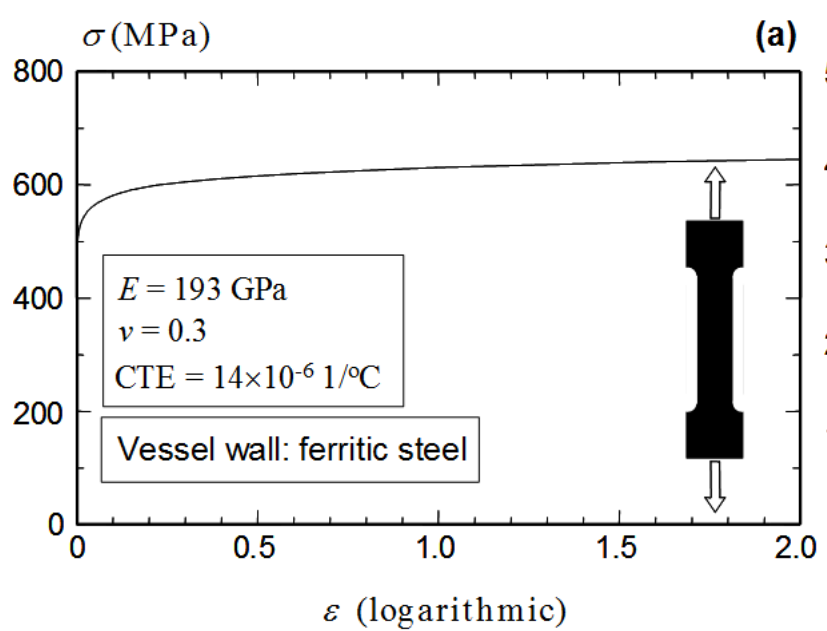

$\sigma(\mathrm{MPa})$

(b)

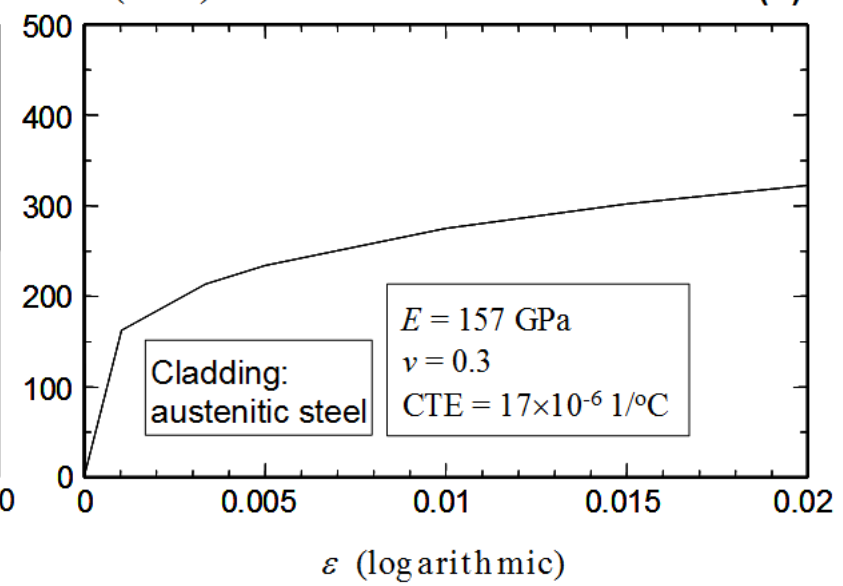

Figure 3 Room temperature, uniaxial, true stress-logarithmic strain relationships for: (a) the vessel wall (ferritic) steel characteristic of ASTM A533B; and (b) the austenitic stainless steel for the cladding.

\subsection{PTS Transient}

The current study considers a critical pressurized thermal shock (PTS) transient characterized by significant changes in both internal pressure and temperature of the coolant in contact with the inner surface of the RPV. This selected transient derives from a risk assessment program initiated by the Sandia National Laboratory to identify critical transients that create potential risks to the operation of RPVs [30]. Figure 4 illustrates the variation of internal pressure and coolant temperature with respect to time, computed by the RELAP5 code for the analysis of thermal-mechanical transients during accidental events [31]. In a previous study [29], this transient generates a more critical crack driving force, $K_{J}$, than other plausible transients. Simple linear interpolation of these cooling temperatures and the internal pressures yields the smoothed thermal and mechanical loading histories for application in the fracture mechanics models. For the selected transient shown in Fig. 4, the coolant temperature decreases markedly from $286^{\circ} \mathrm{C}$ at $t=0$ to $43^{\circ} \mathrm{C}$ at $t=18 \mathrm{~min}$. The internal pressure decreases from $15.9 \mathrm{MPa}$ at $t=0$ to $0.4 \mathrm{MPa}$ 
at $t=20 \mathrm{~min}$, and then remains at a constant value of $1 \mathrm{MPa}$ for $t>21 \mathrm{~min}$. The computational models described here impose a spatially uniform temperature of $286^{\circ} \mathrm{C}$ and an internal pressure of $15.9 \mathrm{MPa}$ at $t=0$.
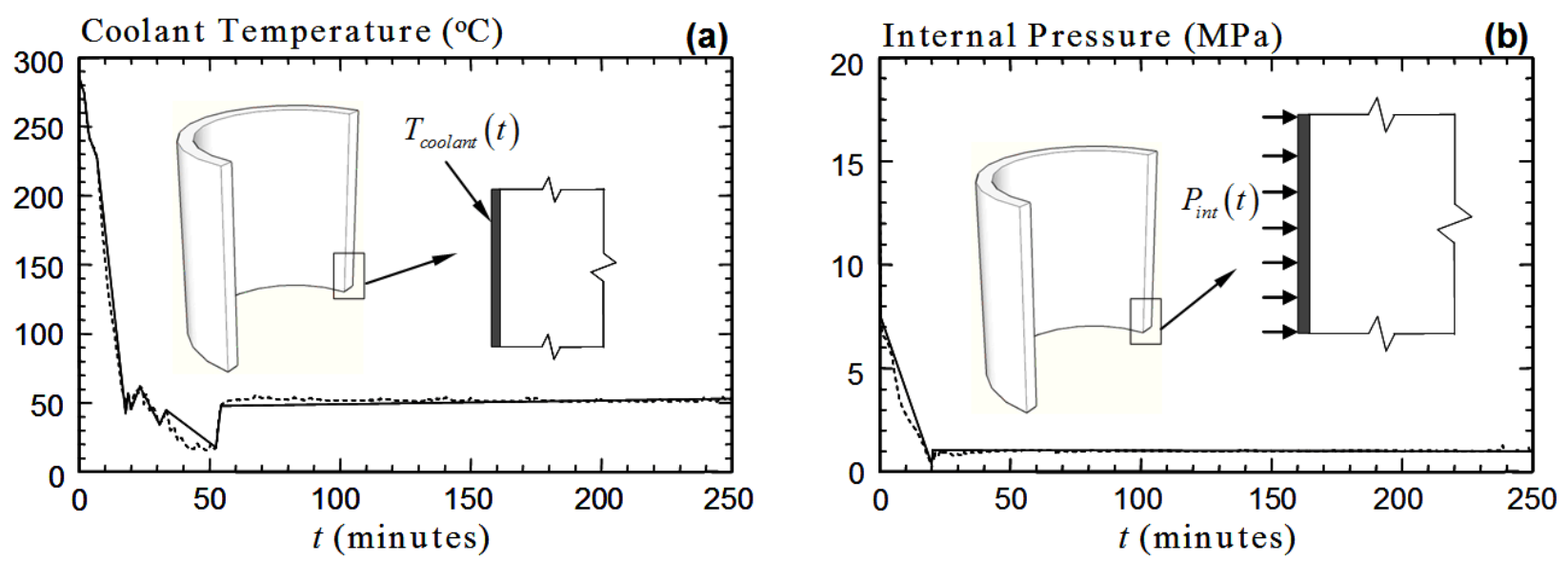

Figure 4 The PTS transient condition: (a) coolant temperature; and (b) internal pressure. Linear interpolations, indicated by the solid line, smooth these loading histories for input to the fracture analysis.

Based on the coolant temperature histories shown in Fig. 4(a), the loading module in the fracture analysis

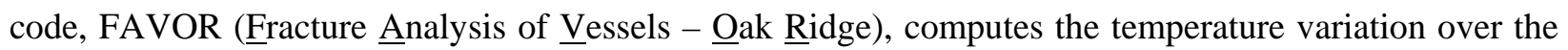
wall thickness of the RPV for the entire transient history. The FAVOR code [4, 5], developed and maintained by the Oak Ridge National Laboratory, performs both probabilistic and deterministic (linearelastic) fracture analysis for reactor pressure vessels subjected to PTS and other thermal-mechanical events. FAVOR computes the temperature profile through the wall of an RPV over the time history of an event by solving the transient heat conduction equation for a 1-D axisymmetric finite element model of the combined cladding and base materials in the vessel wall. Figure 5 shows the variation of temperature over the normalized wall thickness, $t_{\text {nom }}=\left(r-r_{\text {int }}\right) / t_{\text {wall }}$, at a time interval of 5 minutes. The thermal transient shows pronounced temperature decreases near the inner surface $\left(t_{\text {nom }} \rightarrow 0\right)$ of the RPV for $t>10 \mathrm{~min}$. The marked temperature decrease creates significant temperature gradients over material surrounding the elliptical crack located very near the inner surface of the RPV (see Fig. 2), and thus elevates crack-front stresses.

\subsection{Finite Element Models}

Preliminary analyses [29] revealed the inability of 8-node isoparametric (brick) elements to capture accurately the complex, nonlinear deformations in the crack front region under the combined thermal- 
pressure loading. The 8-node elements also failed to predict the $K_{I}$ variation along the crack front under linear-elastic conditions specifically for the thermal gradients imposed in these studies. The adoption of 20-node isoparametric elements with reduced integration for the entire, quarter model of the RPV, as shown in Fig. 6, proved sufficiently accurate in a detailed mesh convergence study.
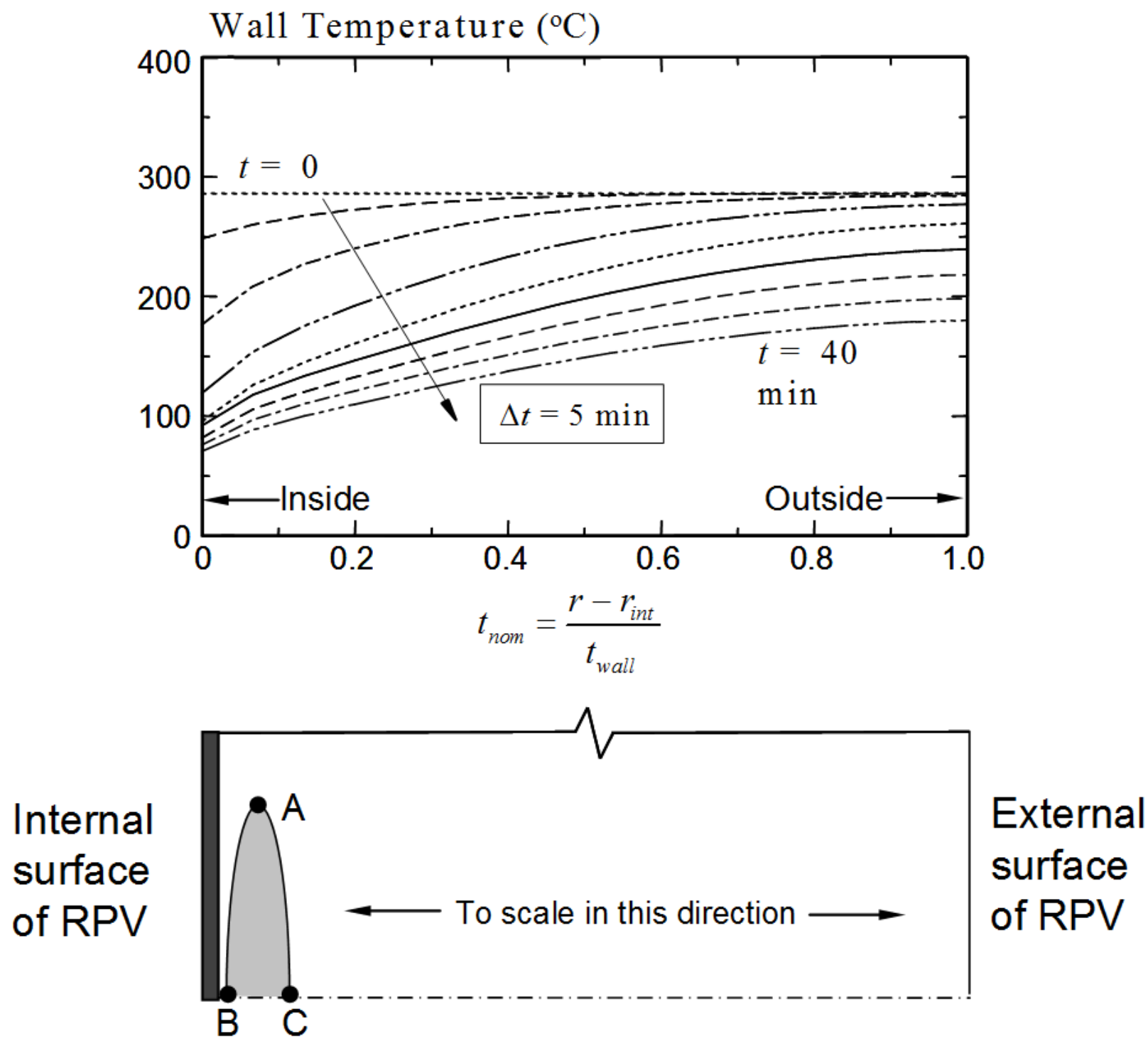

Figure 5 Temperature variations over the wall thickness at time intervals of 5 minutes computed by the 1-D axisymmetric analysis in FAVOR. 


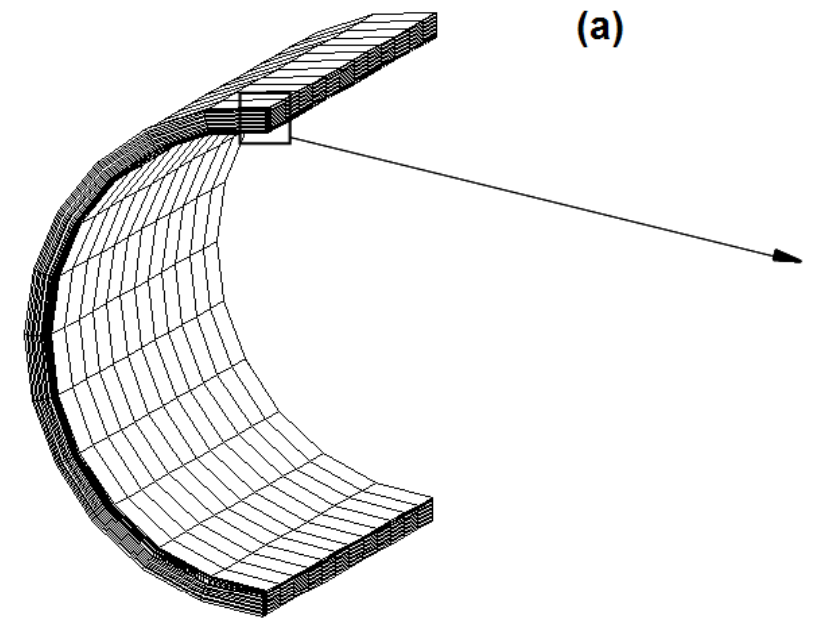

(d)
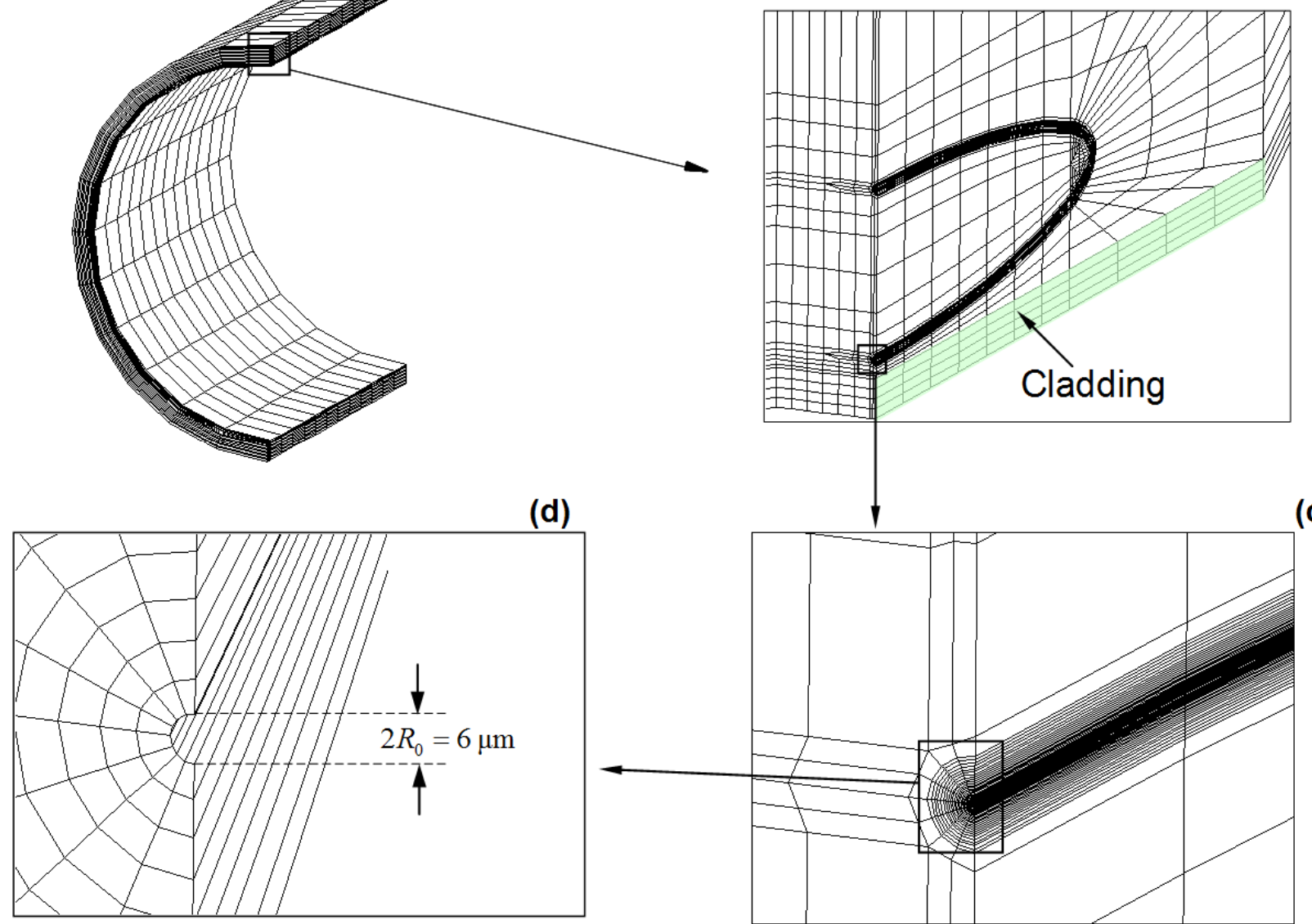

(c)

Figure 6 The quarter-symmetric finite element model using 20-node brick elements for an RPV with an axially embedded elliptical flaw: (a) global model; (b) close-up view for the embedded crack with 30 elements along the semi-elliptical crack front; (c) each crackfront position has 31 rings of elements; and (d) the initial root diameter of the crack front equals $6 \mu \mathrm{m}$.

The presence of two planes of symmetry permits the use of a quarter-symmetric model. The use of an advanced, 3-D crack mesh generator, FEA-Crack [32], coupled with the mesh building-manipulation capabilities in MSC-Patran greatly streamlined and simplified the mesh construction process. This procedure builds a detailed finite element model having a continuous transition from the crack-front mesh to the adjacent global mesh using only 20-node brick elements. The half-perimeter of the elliptical crack represented in the mesh contains 30 elements along the curved front, with 31 rings of elements surrounding each crack-front node. The first ring of elements defines an initial root radius of $3 \mu \mathrm{m}$ to facilitate numerical convergence for the finite strain solutions early in the loading. The finite element model shown in Fig. 6 has 92,240 nodes and 20,800 (20-node) elements, with 4 layers of elements defined over the inner cladding steel. The model imposes full continuity on the interface between the cladding steel and the base material (i.e., the elements in the base material and those in the cladding share the same nodes on the base-cladding interface). The model constrains the out-of-plane displacements on 
the planes of symmetry. A time-dependent pressure applied on the inner surface of the vessel simulates the mechanical transient indicated in Fig. 4(b). A simple code provides the temperature value at each node of the 3-D RPV model for a time interval of $\Delta t=1 \mathrm{~min}$, based on the variation of temperature over the wall thickness of the RPV shown in Fig. 5. Linear interpolations define the temperature values for nodes in between the points where temperature values are computed for the 1-D axisymmetric model in FAVOR.

The computation of accurate Weibull stress values requires a significantly higher level of crack-front mesh refinement than is needed to compute just the elastic-plastic $J$-values. The Weibull stress value depends very strongly on the computed stresses at the peak stress location ahead of the blunting crack front. Computation of elastic-plastic $J$-values, however, employs domain-energy integrals evaluated largely over elements more distant from the crack front. For the RPV described above, a FE mesh with 65,800 nodes and 14,900, 20-node elements (10 rings of elements surrounding each crack-front location) provides identical elastic-plastic $J$-values to those from the very refined model (with 31 rings of elements surrounding each crack-front location) presented in Fig. 6. An even more less-refined model very likely yields accurate $J$-values but was not investigated in this study.

Finite element analyses of the 3-D models described above and analyses of the 2-D models described in subsequent sections are performed using the fracture mechanics research code WARP3D [31]. The Mises constitutive model with $J_{2}$ flow theory describes the nonlinear material response and element behavior follows the finite-strain formulation. An independent post-processing code computes the Weibull stress values based on the crack-front stress/strain fields obtained from the WARP3D analyses. The computation of Weibull stresses reflects the "history" effect whereby the probability of cleavage fracture does not decrease over the loading history, and therefore prohibits reductions in the computed Weibull stress in the event of unloading. The numerical procedures integrate the effective stress ( $\sigma_{1}$ in the current study) over the deformed volume of the fracture process zone, defined by a stress cutting parameter $\lambda=2$ (i.e., $\sigma_{1} \geq 2 \sigma_{0}$ ) to yield the Weibull stress values.

\subsection{Crack-Front Constraint}

Figure 7 shows the variation of $K_{I}, T$ and $\beta$ values along the crack front for three different points in time of the PTS transient ( $t=0,10 \mathrm{~min}$ and $18 \mathrm{~min}$ ) computed from a linear-elastic analysis of the very refined, 3-D model of 20-node elements. $K_{I}, T$-values are extracted using an interaction integral approach [33,34] which adopts a superimposed equilibrium state consisting of the actual fields and auxiliary fields of displacements, stresses and strains. The auxiliary fields, corresponding to plane-strain $K_{I}$ and $T$-stress, enable direct calculation of $K_{I}$ and $T$-values for the actual, 3-D physical fields. The 
analyses reveal a maximum $K_{I}$-value at location B on the crack-front, the point nearest to the inner surface of the RPV. Between points B and A, the $K_{I}$-values decrease and reach a minimum value on the crack front at point A. The $K_{I}$-values increase again between points $\mathrm{A}$ and $\mathrm{C}$. The high temperature gradient over the wall thickness caused by the thermal transient at $t=10$ and 18 min continues to increase the $K_{I}$-values along the crack front, with the maximum $K_{I}$-values over the entire transient achieved at $t=18 \mathrm{~min}$.
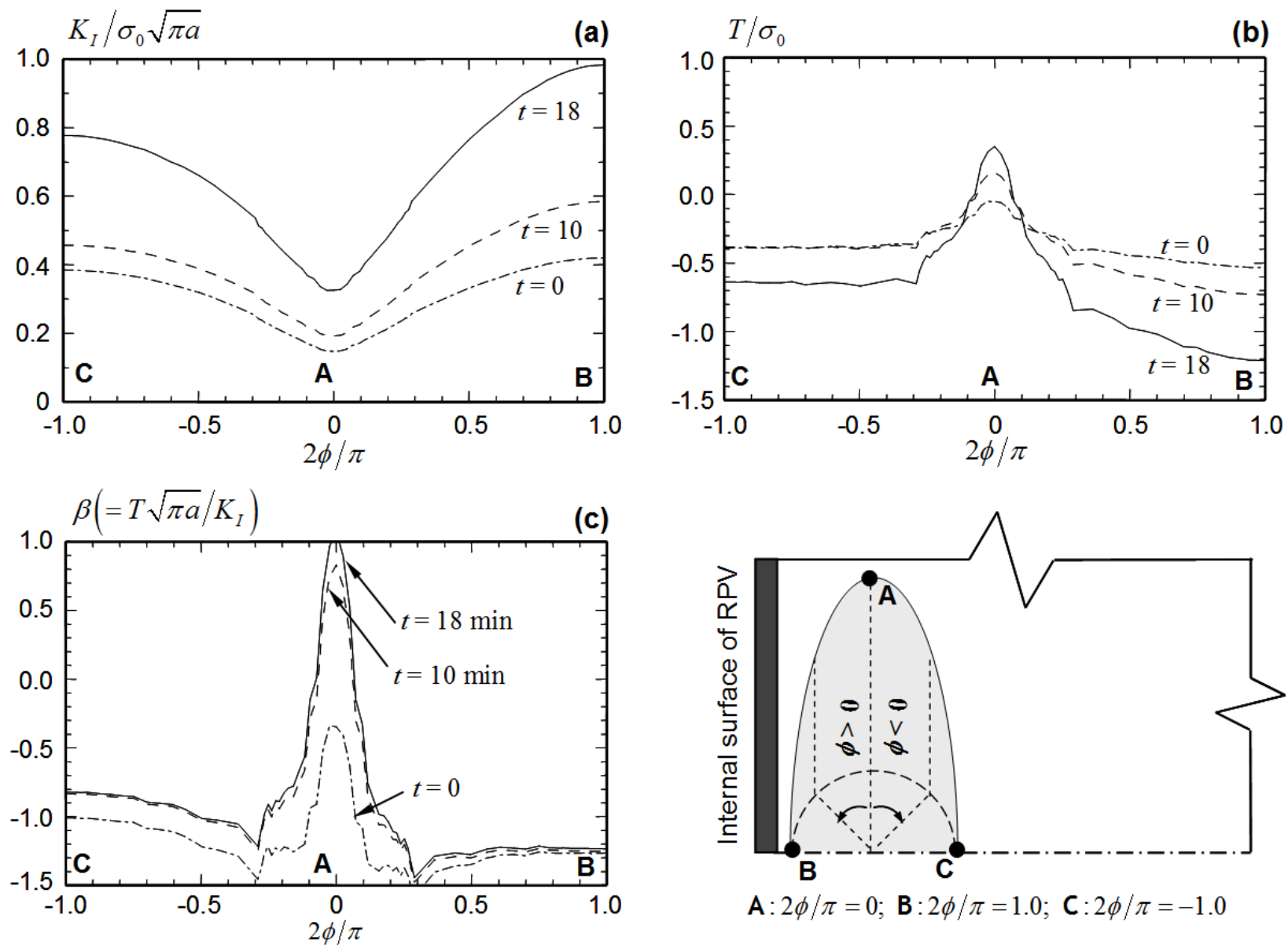

Figure 7 (a) Variation of the linear-elastic stress intensity factor; (b) variation of the linear-elastic $T$-stress; and (c) variation of the non-dimensional $\beta$ ratio.

On the basis of a linear-elastic analysis that considers only the effect of $K_{I}$, the critical crack-front location becomes point $\mathrm{B}$, which has the highest $K_{I}$-value and the relatively lowest temperature, see Fig. 5. The Weibull stress assessment described in the following section, which includes the effects of stress redistribution caused by plastic deformation and the corresponding effects on constraint, reveals that point $\mathrm{C}$ and adjacent material becomes the critical region on the crack front. 
The elastic $T$-stress provides a first-order characterization for the levels of stress triaxiality within well contained, localized plastic deformation along the crack front. Negative T-stress configurations, and locations with $T<0$ along a 3-D crack front, experience lower crack-front triaxiality leading to often significant reductions of the opening-mode stress below the levels experienced in common (high constraint) fracture test specimens, e.g., $\mathrm{C}(\mathrm{T})$ and deep-notch $\mathrm{SE}(\mathrm{B}) \mathrm{s}$. Positive $T$-stress configurations and crack front locations with $T>0$ maintain the triaxiality and opening-mode stress levels found in high constraint test specimens. The analyses here show a significant variation of $T$-stress over the crack front. For the internal pressure only which acts at $t=0$, the entire crack front experiences negative $T$ stresses indicating low crack-front constraint conditions. Once the thermal transient imposes a pronounced temperature gradient over the wall thickness (e.g. at $t=10 \mathrm{~min}$ and $18 \mathrm{~min}$ ), the negative $T$ stresses continue to decrease (more negative) in magnitude near points B and C. For the crack-front point A, the differential contraction in the longitudinal direction of the RPV caused by the thermal transient creates a relatively high positive $T$-stress, which maintains high constraint levels at the two ends of the elliptical crack, as shown in Fig. 7(b).

The non-dimensional biaxiality ratio ( $\beta=T \sqrt{\pi a} / K_{I}$ ), shown in Fig. 7(c) for $t=0,10$ min and 18 min, reflects the first-order evolution of constraint conditions along the crack front under increasing $K_{I}$ and $T$ stress fields caused by the PTS transient. The decrease of internal pressure, coupled with the increase of temperature gradient over the wall thickness from $t=0$ to $t=10 \mathrm{~min}$, leads to higher levels of crackfront constraint (less negative and higher positive values of $\beta$ ). The further increase in temperature gradient beyond $t=18$ min does not cause a significant change in the $\beta$-value compared to $t=10 \min$. Figure 7(c) demonstrates the very high crack-front constraint around point A and the low constraint near both points B and C. Notice that the crack-front region near point B (which the largest $K_{I}$-value on the front) also experiences more constraint loss compared to the crack-front region near point C.

\subsection{Weibull Stress Values for the 3-D RPV model}

Procedures for preliminary engineering assessment adopt simplified approaches to compute the cumulative probability of fracture. A simplified Weibull stress approach, as discussed by Wasiluk et al. [29], utilizes a single value of the Weibull stress computed over the volume of material along the entire crack front as the crack-front loading parameter for each time during the transient. The microscale material toughness, $\sigma_{u}$, then takes on the lowest value along the crack front, corresponding to the location with the lowest temperature (point B for the embedded flaw in Fig. 5). Figure 8(a) shows these "total" Weibull stress values computed for the entire crack front in the RPV model using three different values of the Weibull modulus $m(=10,15$ and 20). The sharp reduction of internal pressure at the onset of the PTS transient (as shown in Fig. 4(b)) coupled with relatively mild thermal gradient over the wall 
thickness for $0<t<9$ (as shown in Fig. 5) relaxes the stress levels along the crack front. However, the Weibull stress value remains at a constant value due to the history effect up to $t=9$ min (the history effect prohibits Weibull stress values from decreasing below a previously maintained maximum value). For $t>9 \mathrm{~min}$, the more pronounced thermal gradient over the wall thickness elevates Weibull stress values in the RPV. Figure 8(a) shows the Weibull stress values up to $t=18 \mathrm{~min}$, at which time the Weibull stress attains its maximum value for the considered PTS transient and remains constant beyond $t=18 \min$.
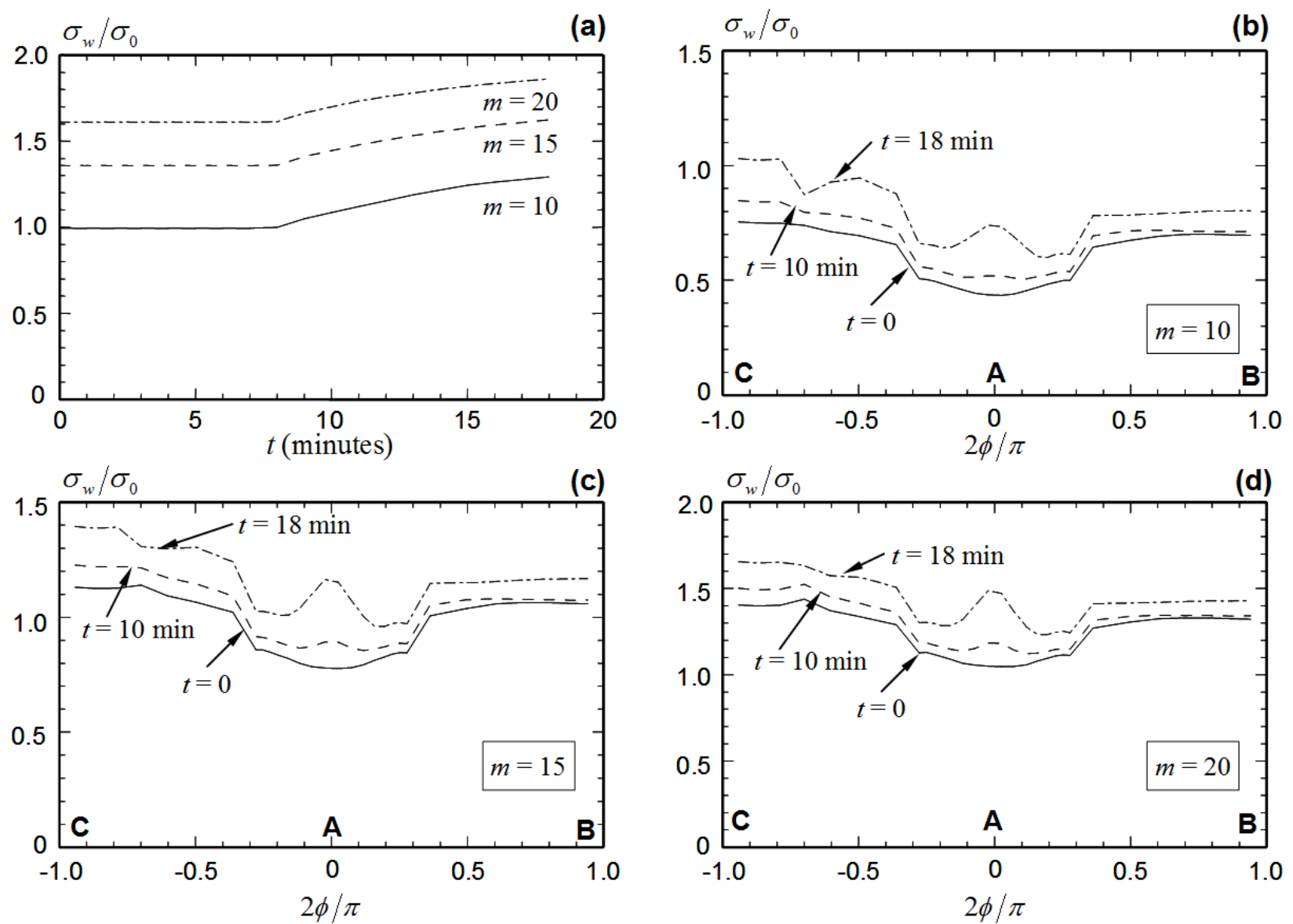

Figure 8 (a) Evolution of the Weibull stress values computed over the entire perimeter of the crack for the transient history; and the variation of local (pointwise) Weibull stresses at $t=0,10$ min and 18 min over the crack front for (b) $m=10$; (c) $m=15$; and (d) $m=20$.

A more refined integrity assessment procedure of the RPV employs Eq. 7, which requires pointwise (or over small segments) values of the Weibull stress at locations along the entire crack front. The numerical procedure used here divides the half-perimeter of the elliptical crack front represented in the model into 30 segments and computes the (local) Weibull stress values within each segment following Eq. 3. Figures 8(b)-(d) compare the Weibull stress variation along the crack front at three times over the transient history, $t=0,10 \mathrm{~min}$ and $18 \mathrm{~min}$, respectively. The maximum value of Weibull stress occurs at the 
crack-front location furthest from the inner surface of the RPV, denoted as point $C$ in Fig. 5; this location also experiences the highest temperature along the crack front. The Weibull stress at crack-front point B, which has the lowest temperature and thus the lowest material toughness, has a lower value compared to the $\sigma_{w}$-value at point $\mathrm{C}$. The maximum value of the local crack driving force represented by $\sigma_{w}$ occurs at point $\mathrm{C}$, which does not correspond to the maximum value of macroscopic crack driving force $\left(K_{I}\right)$ along the crack front as indicated in Fig. 7(a). The less negative value of the $T$-stress at point $\mathrm{C}$, compared to that at point $\mathrm{B}$, imposes a higher constraint on the plastic flow and thus maintains higher stress levels near point C. As indicated earlier in Fig. 1(d), the lower Weibull stress values at point B fall below the threshold Weibull stress value computed from a constant $K_{\min }=20 \mathrm{MPa} \sqrt{\mathrm{m}}$. Therefore, point B does not contribute to the total probability of fracture, even though the local material toughness, $\sigma_{u}$, is the lowest along the entire crack front. Thermal-pressure transients with other characteristics may cause the $\sigma_{w}$-value at point B to increase above $\sigma_{w-\min }$, and thus the contribution to the total probability of fracture at point B may become more significant than the contribution from point C.

The Weibull stress near the two ends of the crack-front (point A) has quite small values in the initial stage of the transient ( $t=0$ and $t=10$ min in Figs. 8(b)-(d)), where $K_{I}$ also remains very small. As $K_{I}$ increases over the transient history, the strongly positive $T$-stress (shown in Fig. 7(b)) at point A ensures well-contained plasticity and continued high levels of stress triaxiality around the crack front. Consequently, the Weibull stress at point A corresponding to $m=20$ (Fig. 8(d)) attains a slightly larger value than the $\sigma_{w}$-value at point $\mathrm{B}$, even though the $K_{I}$-value at point $\mathrm{A}$ is significantly smaller than that at point B, as indicated in Fig. 7(a).

Larger $m$-values ( $>12-15)$ promote strongly the relative contribution of material right at the peak stress location ahead of the blunting crack front to the Weibull stress value. This minimizes any dependence of the $\sigma_{w}$-value on the stress cutting parameter, $\lambda[18,29]$. Wasiluk et al. [26], for example, calibrate an $m$ value of 18-20 for the Euro RPV material (ASTM A508 steel) based on an extensive experimental study conducted by laboratories in European countries [27, 28]. The correctness of Weibull stress values computed using these larger $m$-values depends strongly on the accuracy of high stress values predicted in the peak stress region just ahead of the blunting front. Meshes of 20-node elements with the refinement level employed here seem necessary to achieve converged Weibull stress values in the RPVs for the combined pressure-thermal loadings. The Weibull stress values also reveal some sensitivity to details of the specified true stress - logarithmic strain curve for the base material given the large plastic strain levels that exist immediately behind the peak stress location. 


\section{2-D Modeling Frameworks and Results}

The Weibull stress results presented in the above section provide a set of high-quality, reference values to benchmark several proposed 2-D analysis procedures. The simplified 2-D models offer far greater computational efficiency together with reduced model construction effort. The refined 3-D, nonlinear analyses remain impractical for use in Monte Carlo style simulations increasingly employed to examine the stochastic effects of crack size, shape, location, material properties and PTS transients on vessel integrity.

This section describes several 2-D frameworks to approximate the crack front conditions at key locations (e.g., B and C) for the axially-oriented, embedded flaws considered as critical cases for fracture assessment in RPVs. The objective is to identify a suitable 2-D modeling approach that enables accurate computation of Weibull stress values for the embedded RPV crack under different crack-front constraint conditions and subjected to thermal-mechanical loading transients.

\subsection{Modified Boundary Layer Model}

The leading two terms in the classical William's solution [35], shown here in Eq. 8, demonstrates the similitude in the linear-elastic, crack-front stress fields for crack fronts subjected to identical $K_{I}$ and $T$ stress values,

$$
\sigma_{i j}(r, \theta)=\frac{K_{I}}{\sqrt{2 \pi r}} f_{i j}(\theta)+T \delta_{1 i} \delta_{1 j}
$$

where $\delta_{i j}$ represents the Kronecker delta with the subscript $i$ and $j$ range over $(1,2)$. Subscript one defines the axis perpendicular to the crack front and parallel to the crack plane. The function $f_{i j}(\theta)$ defines the angular variation of the singular stress field characterized by $K_{I}$. The second term in Eq. 8, the elastic $T$ stress, represents a constant compressive or tensile stress field parallel to the crack plane over the asymptotically small region surrounding the crack tip. As discussed previously, the magnitude and sign of $T$-stress affects the stress triaxiality within the plastic zone ahead of the crack tip under small-scale yielding conditions and consequently the stress triaxiality near the crack front.

The conventional modified boundary layer (MBL) model consists of a planar, semicircular region of material loaded directly under remote $K_{I}-T$ displacement fields. Under certain conditions, this model 
provides accurate descriptions displacement-strain-stress fields along 3-D crack fronts. Figure 9(a) shows the configuration of the MBL model subjected to a remotely imposed displacement field computed from the linear-elastic $K_{I}-T$ values under the assumption of plane-strain behavior,

$$
\begin{gathered}
u(R, \theta)=K_{I} \frac{1+v}{E} \sqrt{\frac{R}{2 \pi}} \cos \left(\frac{\theta}{2}\right)(3-4 v-\cos \theta)+T \frac{1-v^{2}}{E} R \cos \theta \\
v(R, \theta)=K_{I} \frac{1+v}{E} \sqrt{\frac{R}{2 \pi}} \sin \left(\frac{\theta}{2}\right)(3-4 v-\cos \theta)-T \frac{v(1+v)}{E} R \sin \theta
\end{gathered}
$$

where $E$ denotes the Young's modulus of the material and $v$ refers to the Poisson's ratio. The geometric parameter $R$ measures the distance from the crack tip to the edge of the boundary layer model and $\theta$ defines the counter-clockwise angle from the horizontal $x$-axis as shown in Fig. 9(a). The radius $R$ of the MBL model here equals $2.5 \mathrm{~m}$, with the thickness of the model defined as $25 \mathrm{~mm}$. The model shown in Fig. 9(a) has an initial root radius of $3 \mu \mathrm{m}$ to facilitate numerical convergence of the finite-strain solutions early in the loading. The crack-front mesh in the MBL model has the same number of elements in the circumferential $(\theta)$ direction and similar crack-front element sizes as those in the refined 3-D RPV model. The FE model in Fig. 9(a) utilizes 840 20-node brick elements and 6450 nodes, with out-of-plane displacements constrained to impose plane-strain conditions. The material properties follow the uniaxial stress-strain relationship shown in Fig 3(a) for the base material.

Figures 9(b)-(d) compare the (opening mode) crack-front stress, $\sigma_{y y}$, computed on $y=0$ from the planestrain MBL model with stress fields at locations A, B and C of the 3-D crack front for two internal pressure $\left(P_{\text {int }}\right)$ levels. The remote displacement loadings imposed on the MBL models derive from Eqs. 9 and 10 using $K_{I}$ and $T$-values at points A, B and C computed from the linear-elastic analysis of the 3-D RPV model. The horizontal axis in Figs. 9(b)-(d) measures the distance from the crack tip with respect to $J / \sigma_{0}$, where the opening displacement of the blunted crack tip is $\approx J /\left(1.5 \sigma_{0}\right)$ [36]. The pressure $P_{0}$ in Fig. 9 denotes the initial pressure level at $t=0$ of the transient as shown in Fig. 4(b), which generates relatively low stress fields near the crack front. To assess the MBL model for higher pressures and thus more extensive plasticity in the RPV, the analyses also impose $P_{\text {int }}=2 P_{0}$. At the smaller load level of $P_{\text {int }}=P_{0}$, the stress field near point A, with $K_{I}=10.7 \mathrm{MPa} \sqrt{\mathrm{m}}$ and $T / \sigma_{0}=-0.06$, has not yet reached a self-similar solution as indicated by the peak stress location at a distance $x /\left(J / \sigma_{0}\right) \approx 10$ ahead of the crack tip (i.e., the $3 \mu \mathrm{m}$ initial root radius in the model continues to influence the stress fields). As the internal pressure increases to $2 P_{0}$, point A experiences a higher stress field with the linear-elastic $K_{I}$ and 
T-stress values doubled. The 3-D RPV model and the plane-strain, MBL model now show closer agreement in $\sigma_{y y}$-values computed near location A and with the peak stress at $x /\left(J / \sigma_{0}\right) \approx 2$.

(a)
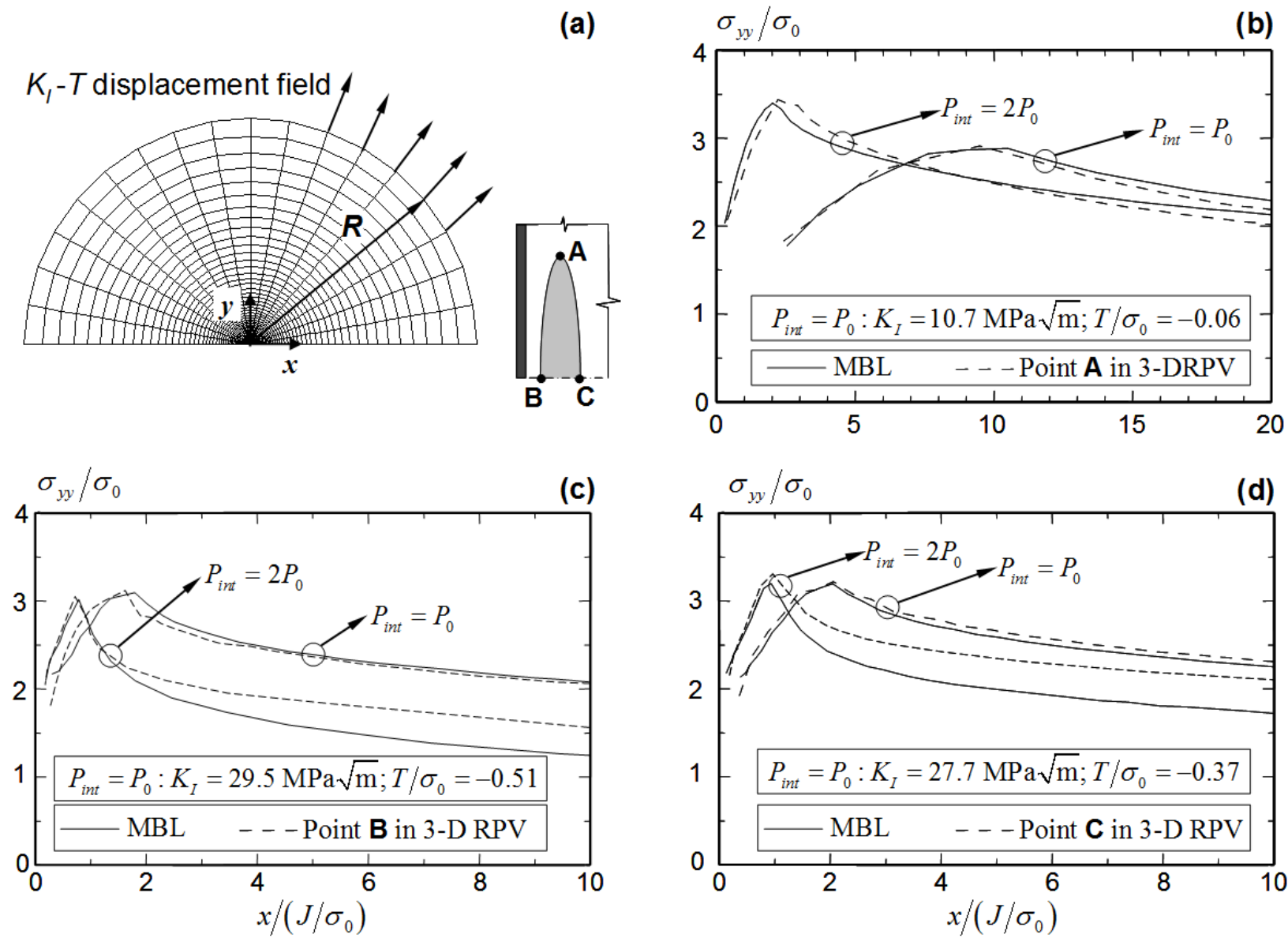

Figure 9 (a) Configuration of the modified boundary layer (MBL) model; and crack-front stresses at $P_{\text {int }}=P_{0}$ and $P_{\text {int }}=2 P_{0}$ for (b) point $\mathrm{A}$; (c) point B; and (d) point $\mathrm{C}$; along the 3-D curved crack front.

The crack-front locations B and C experience significant constraint loss compared to location A, as shown by the more negative $T / \sigma_{0}$ values listed in Figs. 9(c) and 9(d). At the smaller internal pressure, $P_{\text {int }}=P_{0}$, crack-front stresses in the MBL model agree very well with stresses from the 3-D RPV model. As the internal pressure increases to $P_{\text {int }}=2 P_{0}$, the $T$-stress at location $\mathrm{C}$ approaches the yield stress of the base metal while at $\mathrm{B}$ the (linear-elastic) estimate of the $T$-stress exceeds the yield stress of the base metal both values indicating the potential development of large-scale yielding. The peak stress location moves characteristically nearer to the blunted tip for reduced constraint, and the crack-front stress fields obtained from the MBL model now fall much lower than 3-D RPV stresses ahead of the peak stress location at both $\mathrm{B}$ and $\mathrm{C}$. The large negative $T$-stress near locations $\mathrm{B}$ and $\mathrm{C}$ effectively invalidates adoption of the MBL to compute Weibull stress values for all but relatively low internal pressures (and $K_{I}$-values). 
The internal pressure acting on the cylindrical shape of the RPV creates a stress gradient over the wall thickness. The radial and circumferential stresses for a very long (or approximately plane-strain) cylindrical model subjected to internal pressures derive from plane elasticity as,

$$
\begin{gathered}
\sigma_{r r}=P_{i n t} \frac{r_{i n t}^{2}}{r_{i n t}^{2}-r_{e x t}^{2}}\left(1-\frac{r_{e x t}^{2}}{r^{2}}\right) \\
\sigma_{\theta \theta}=P_{i n t} \frac{r_{i n t}^{2}}{r_{i n t}^{2}-r_{\text {ext }}^{2}}\left(1+\frac{r_{\text {ext }}^{2}}{r^{2}}\right)
\end{gathered}
$$

where $\sigma_{r r}$ denotes the radial stress and $\sigma_{\theta \theta}$ defines the circumferential stress. The parameters $r_{\text {int }}$ and $r_{\text {ext }}$ refer to the internal and external radius of the RPV. The circumferential stress, $\sigma_{\theta \theta}$, acts as the opening stress on the embedded axial crack shown in Fig. 2(a), while the radial stress contributes to the compressive $T$-stress near the crack-front points B and C. For $P_{i n t}=2 P_{0}=31.8 \mathrm{MPa}, \sigma_{\theta \theta}$ varies from $0.76 \sigma_{0}$ at the inner surface of the RPV to $0.68 \sigma_{0}$ at the outer surface of the RPV based on Eq. 12 . These values of local stresses, together with the low plasticity constraint near the embedded crack front, cause significant redistribution of the crack-front stresses to the adjacent material and large-scale yielding.

The "embedded" configuration of the elliptical flaw in the wall of a cylindrical RPV imposes significantly different conditions on the plasticity driven stress redistribution than exist in the MBL model. The idealized geometric configuration of a MBL model, subjected to $K_{I}-T$ loadings, represents a singleended crack of infinite length contained in an infinite, plane-strain plate. As the plastic zone in the MBL model increases in size, the crack-front stress field never interacts with the geometric boundary of the model. The (very) close by inner surface of the RPV and the low yield-strength cladding affect the stress re-distribution near the crack-front point $\mathrm{B}$ as the plastic zone at point B becomes comparable in size with the distance between point B and the inner surface of the RPV. Similarly, the MBL model does not reflect the interaction between the two crack-front stress fields as the plastic zones near points $\mathrm{B}$ and $\mathrm{C}$ of the RPV model grow larger, e.g., at a load level of $P_{\text {int }}=2 P_{0}$. For an "embedded" flaw in a 2-D configuration, represented, for example, by the plane-strain $\mathrm{M}(\mathrm{T})$ model, Wang and Parks [37] found that this difference in stress redistribution near the crack fronts causes significant deviations in the crack-front stress fields of the $\mathrm{M}(\mathrm{T})$ [embedded crack] model from that of the idealized MBL model subjected to the same $K_{I}$ and $T$-stress. Other configurations with only one crack tip, including those with negative $T$ stress, did not reveal this deviation, even to surprisingly high load levels [37]. 


\subsection{Plane-Strain M(T) Model}

An M(T) model likely better represents the plasticity driven stress redistribution of the "embedded" flaw in the RPV based on results of the previous section. Figure 10(a) shows the schematic configuration of an $\mathrm{M}(\mathrm{T})$ model subjected to a remote, uniform axial stress. The $\mathrm{M}(\mathrm{T})$ specimen has a width $2 W$ equal to the wall thickness of the RPV and a height $2 H=8 \mathrm{~W}$. The presence of two planes of symmetry permits the use of a quarter-symmetric model, as shown in Fig. 10(b), which has 1000, 20-node brick elements with 8000 nodes. The crack-front mesh of the M(T) specimen closely matches the mesh of the 3-D RPV model and also has an initial root radius of $3 \mu \mathrm{m}$. The material properties follow the uniaxial stress-strain relationship shown in Fig 3(a) for the base material. Loading of the model occurs through uniform pressure applied on the far end as shown in Fig. 10(a). Constraints on the out-of-plane displacements impose the plane-strain condition.

For internal pressure acting alone on the RPV, the 3-D analyses determine a biaxiality ratio of $\beta=-1.01$ for location $\mathrm{C}$ on the crack front, see Fig. 7(c). Handbook solutions for $T$-stress in standard specimens and several scoping analyses show that an $a / W=0.092$ for this $\mathrm{M}(\mathrm{T})$ specimen provides the same value of $\beta=-1.01$. Figure 10(c) shows excellent agreement of the crack-front stresses, $\sigma_{y y}$, obtained from this $\mathrm{M}(\mathrm{T})$ specimen and those from location $\mathrm{C}$ in the RPV model under the high level of internal pressure, $P_{i n t}=2 P_{0}$. This demonstrates the applicability of the $\mathrm{M}(\mathrm{T})$ model to approximate very accurately the stress redistribution for the embedded flaw in the RPV for a pressure transient.

During the combined pressure-thermal transient, the spatial variation in temperature gradients over the wall thickness of the RPV, shown in Fig. 5, lead to a continuous variation in the $\beta$-value at locations along the crack front, including point $\mathrm{C}$. The $a / W$ selected for the $\mathrm{M}(\mathrm{T})$ specimen must thus change accordingly to maintain the RPV value of $\beta$ in the presence of changing spatial variation of temperature gradient. Consider the PTS transient at $t=18 \mathrm{~min}$. To match the RPV $\beta$-value at location $\mathrm{C}$ at $t=18$ min, the $\mathrm{M}(\mathrm{T})$ specimen must now have $a / W=0.123$. This $\mathrm{M}(\mathrm{T})$ model is loaded with the uniform, remote axial stress until $K_{I}$ and the $T$-stress reach the RPV levels at location C, also at $t=18 \mathrm{~min}$. Figure 10(d) compares the opening-mode stress fields for the M(T) model with those for the RPV model. The comparison clearly is quite poor. 

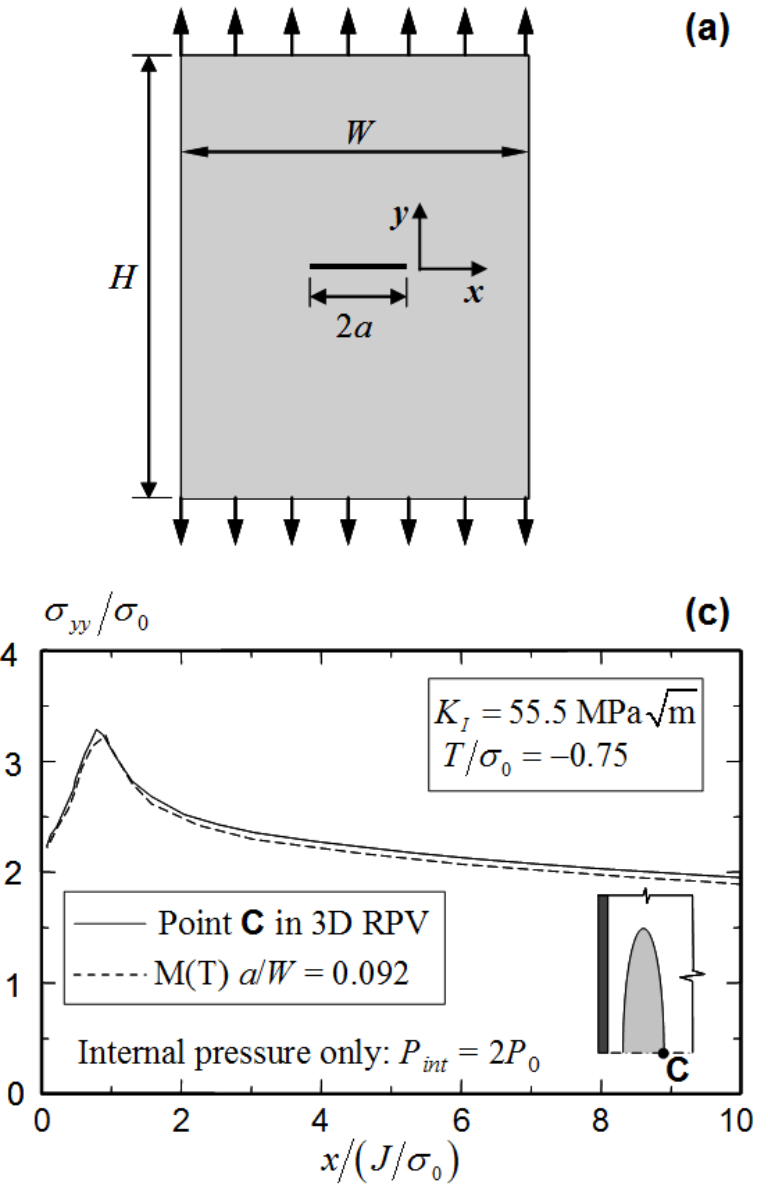

(a)

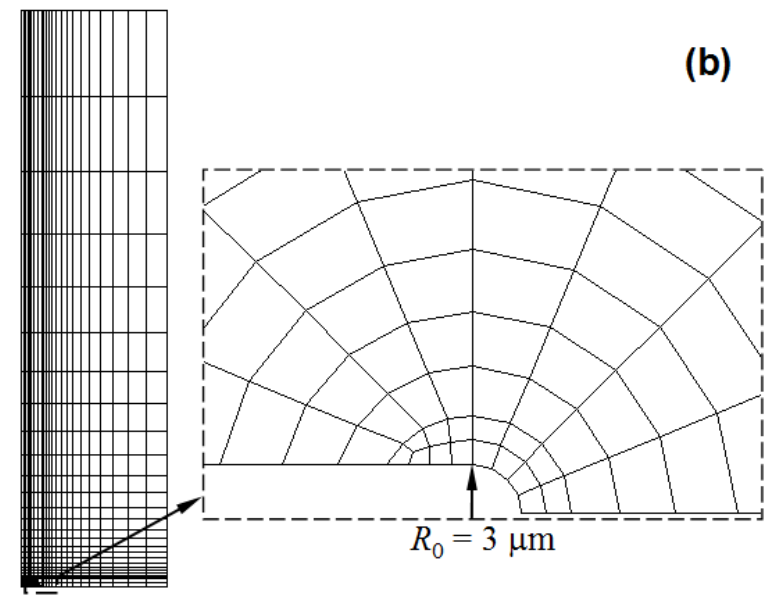

(c)

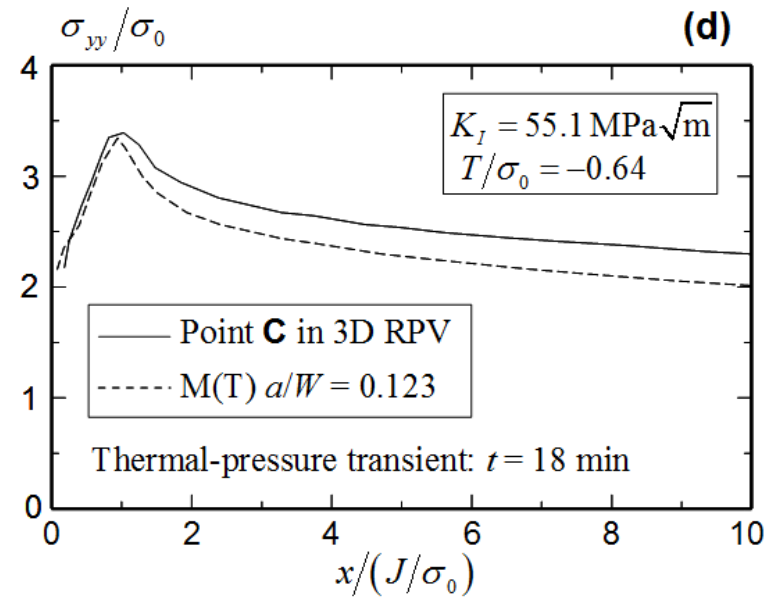

Figure 10 (a) Configuration of the M(T) model; (b) FE mesh for the M(T) model; comparison of the crack-front stress, $\sigma_{y y}$, from the plane-strain $\mathrm{M}(\mathrm{T})$ model and from the embedded crack in the 3-D RPV under: (c) internal pressure only; and (d) combined thermal-pressure transient.

The discrepancy evident in Fig. 10(d) arises from the cumulative effects of plastic deformation and stress redistribution along the crack front in the RPV that occurs under continuously varying constraint levels caused by the changing spatial variation of temperature gradients during the PTS transient. The $\beta$-values provide the first-order characterization of this effect. Figure 11 compares the evolution of $\beta$-values at five locations along the RPV crack front over the transient history. Points B and C correspond to the maximum crack width and where the crack front remains relatively straight (or "flat") in the $\phi$ direction. The crack front locations near points D and E are relatively more "curved" in the $\phi$ direction compared to points B and C, as shown in Fig. 11(c). For crack-front points between B and E with low constraint at $t=0$ (pressure only), the thermal transient increases slightly the crack-front constraint over the time from $t=0$ to $t=18 \mathrm{~min}$. For point A, with initially higher crack-front constraint at $t=0$, the thermal transient increases significantly the crack-front constraint from a small negative $T$-stress at $t=0$ to a large positive $T$-stress at $t=18 \mathrm{~min}$ (recall that $K_{I}$-values are also much lower in this region). As Fig. 10(c) shows 
clearly, the $\mathrm{M}(\mathrm{T})$ model with a fixed $\beta$-value overestimates the constraint loss near point $\mathrm{C}$ of the 3-D curved crack front in the RPV subjected to a thermal-pressure transient.
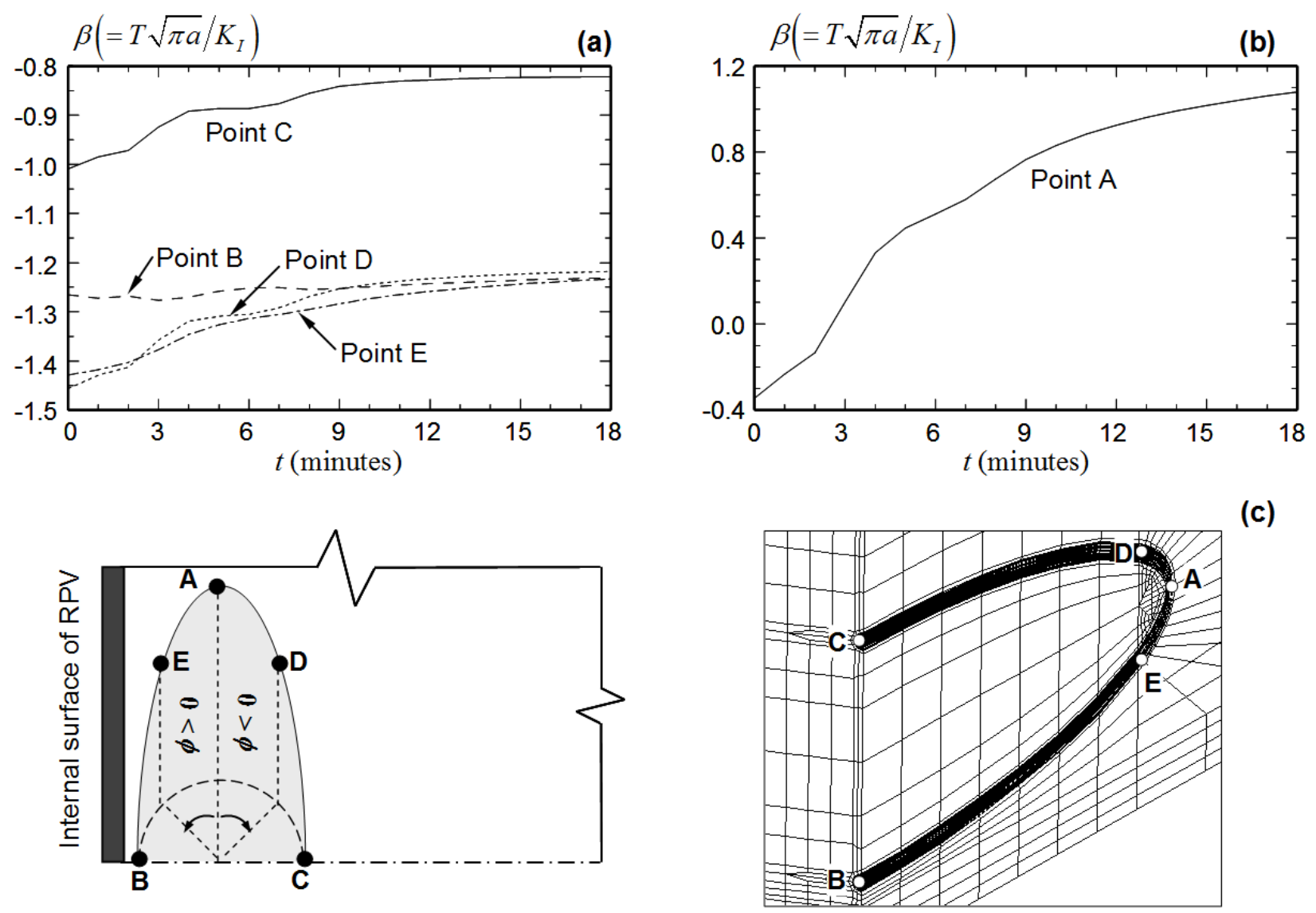

(c)

Figure 11 Evolution of the $T$-stress biaxiality ratio, $\beta$, over the PTS transient history at: (a) points B, $C$, D, and $E$ with low constraints; (b) point A with high constraints; and (c) locations of the different crack-front points.

The plane-strain, $\mathrm{M}(\mathrm{T})$ configuration provides an excellent 2-D model to approximate the crack-front stress fields of the embedded RPV flaw but only for an internal pressure transient. Under this restriction, the RPV $\beta$-values remain invariant of the applied loading history. For the more practical case of combined pressure-thermal transients, the results here show that the $\mathrm{M}(\mathrm{T})$ configuration does provide a suitable 2-D, simplified model.

\subsection{2-D RPV Model}

The detailed 3-D analyses demonstrate that the elongated shape of the axially-oriented, embedded flaw in the RPV $(a / c=0.17$ ) have minimal effect at the mid-height of the crack (points B and C). This leads to consideration of a 2-D, axial cross-section for the cylindrical vessel taken through the center of the RPV 
crack, see Fig. 12(a). The model includes the cladding layer and has an embedded crack of length $2 a$ located at the same through-wall position as the 3-D RPV crack across points B and C. The plane-strain, finite element mesh shown in Fig. 12(a), has one-layer of brick elements extracted from the 3-D RPV model and therefore has exactly the same mesh as the 3-D model for the crack-front locations considered. The equivalent 2-D RPV model shown in Fig. 12(a) has 1110, 20-node elements with 8390 nodes. Constraints on the out-of-plane displacements again impose plane-strain conditions. The material properties follow the two uniaxial, stress-strain curves in Figs. 3(a) and 3(b) for the base material and for the cladding austenitic steel, respectively. The loading consists of the thermal-pressure transient described in Fig. 4. The model requires only a few minutes to compute the full nonlinear solution for the PTS transient and thus becomes feasible for use in the stochastic studies.

(a)
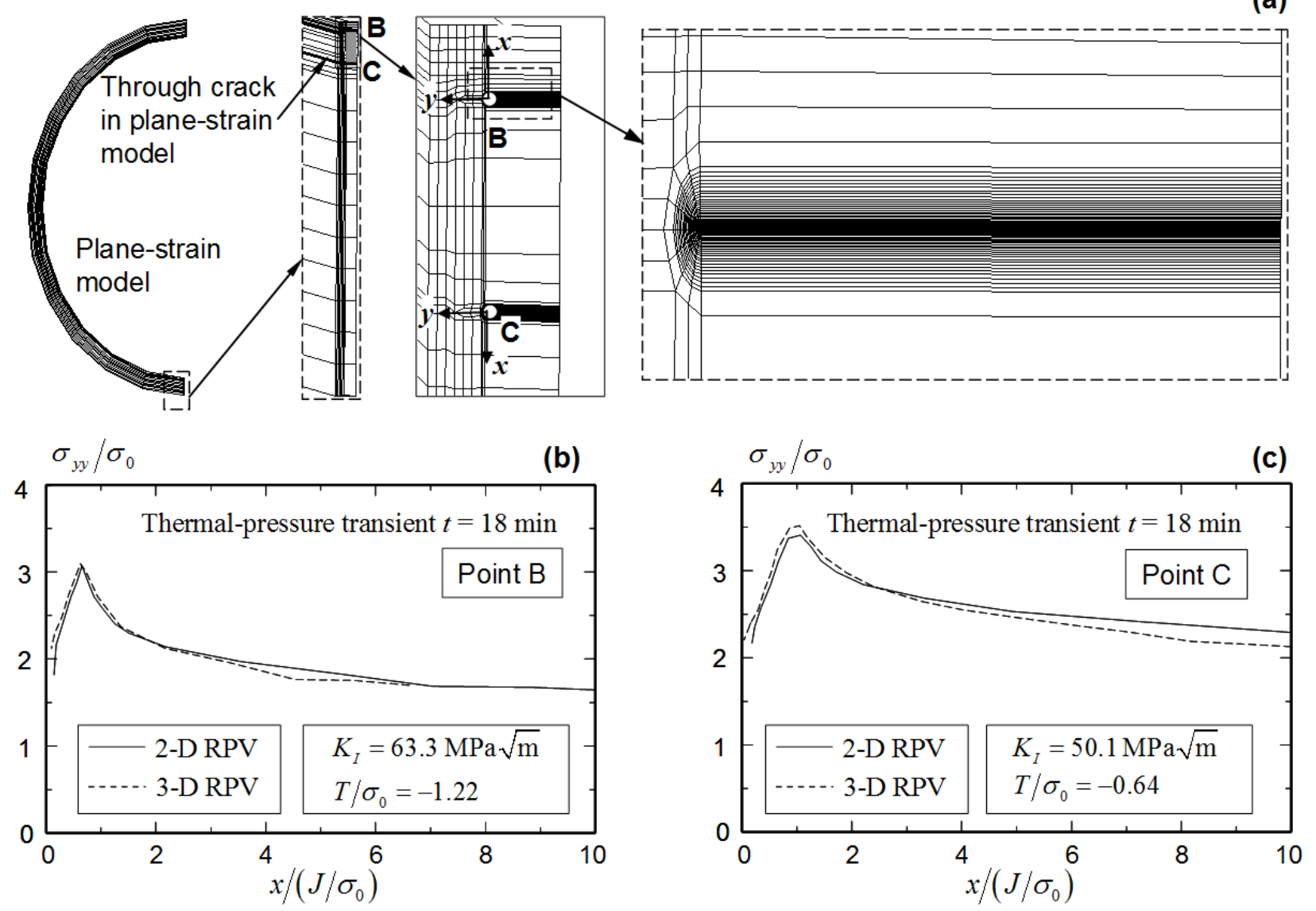

Figure 12 (a) Finite element meshes for a plane-strain, RPV model (actually a 3-D model with onelayer of elements and plane-strain constraints); and comparison of the crack-front stress, $\sigma_{y y}$, computed from the plane-strain RPV model and from the 3-D RPV model: (b) at the crack-front point $B$; and (c) at the crack-front point $C$.

Figures 12(b) and 12(c) compare the crack-front stresses, $\sigma_{y y}$, near points B and C obtained from the simplified, plane-strain RPV model and those from the 3-D RPV model at $t=18$ min in the thermal- 
pressure transient. The crack-front stresses, $\sigma_{y y}$, from the plane strain model for the RPV exhibit very close agreement with those from the 3-D analyses. This plane-strain RPV model thus reproduces (both the in-plane and the out-of-plane) constraint levels near the embedded, elliptical crack in the 3-D RPV model subjected to a thermal-pressure transient. The $g(M)$ function in Eq. 6, calculated from stress fields for this simplified RPV model, then quantifies the effects of constraint loss experienced along the corresponding crack-front in the 3-D RPV model. This plane-strain RPV model proves to be an appropriate and computationally inexpensive, 2-D alternative model to compute the Weibull stress at locations with low constraint along the 3-D embedded crack front.

\subsection{Weibull Stress Approximation Using 2-D Models}

For crack-front locations with low constraint $(\beta<0)$ in an RPV subjected to combined thermal-pressure transients, the Weibull stress values derived from the elastic-plastic analysis of a 2-D (plane-strain) RPV model appear quite accurate. The very similar boundary constraints and the identical loading conditions imposed on the 2-D and 3-D RPV model lead to a quite similar level of constraint loss experienced by locations away from ends of the elliptical crack. The crack-front stress fields near points B and C therefore maintain a similar relationship between the (non-dimensional) opening stress, $\sigma_{y y} / \sigma_{0}$, and the (non-dimensional) crack-tip distance, $x /\left(J / \sigma_{0}\right)$, as shown in Fig. 12. However, adjacent layers of elements in the 3-D RPV model provide additional material to resist the crack-front loading, and therefore reduce the pointwise $K_{J}$-values computed from the 3-D RPV model compared to those from the 2-D RPV model, as demonstrated in Fig. 13. The higher $K_{J}$-values in the 2-D RPV model (20\% higher at points $\mathrm{B}$ and $\mathrm{C}$ of the $3-\mathrm{D}$ RPV model at $t=18 \mathrm{~min}$ ) increases the Weibull stress value, and leads consequently to a conservative estimate of the cumulative probability of fracture. A $20 \%$ overestimation of $K_{J}$-values in this case leads to a $4 \%$ overestimation of the $\sigma_{w}$-value for an $m$ of 20.

With elastic-plastic $K_{J}$-values computed from the 2-D and 3-D RPV models, the following steps detail the procedure to approximate the Weibull stress values near the front in the 3-D RPV model:

1) Compute the constant $\tilde{C}(m)$ from the standard, plane-strain boundary layer model (BLM) with the base metal material properties for $K_{I}(T=0)$ loading with the expression,

$$
\tilde{C}=\frac{\sigma_{w}^{m}}{L_{B L M} K_{B L M}^{4}}
$$


$\tilde{C}$ reaches a constant value as crack-front stresses attain a self-similar state with increased loading of the model. Here, $K_{B L M}=K_{I}$, and $L_{B L M}$ denotes the length of the crack front in the finite element mesh for the boundary layer model (often $L_{B L M}=1$ in FE models using 2-D elements rather than the 1 layer of 3-D elements adopted here)
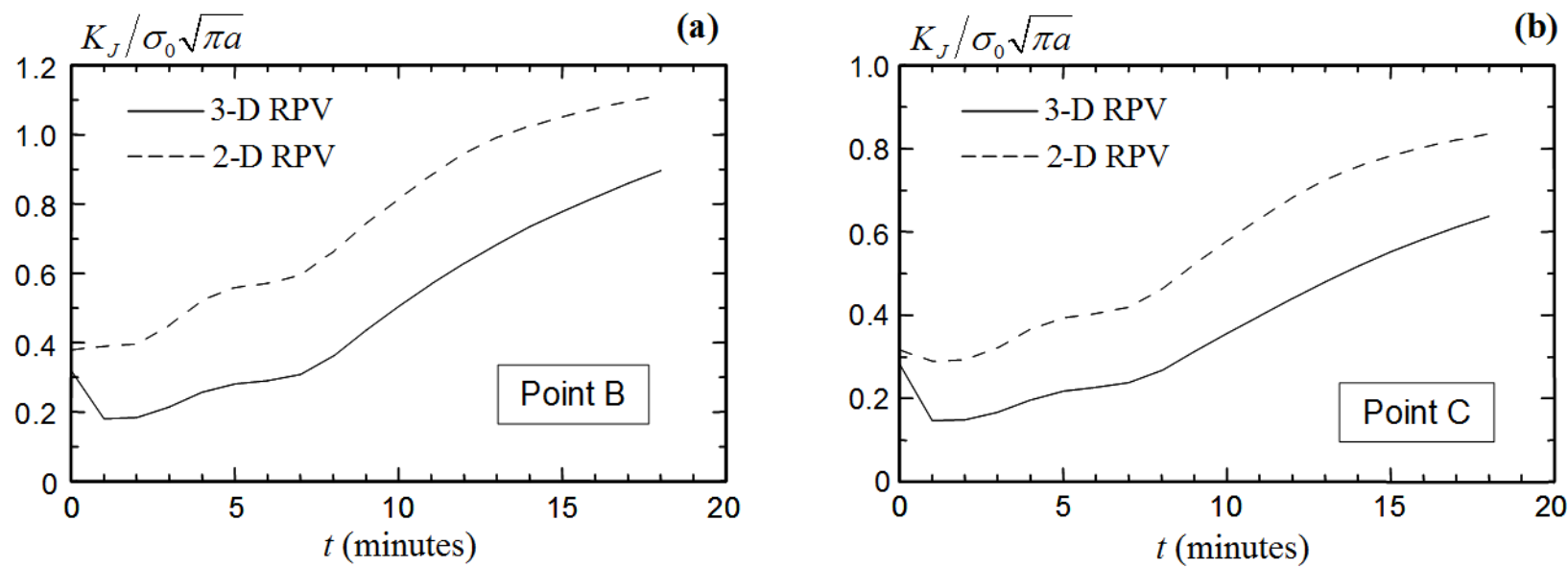

Figure 13 Evolution of the elastic-plastic $K_{J}$-values over the transient history computed from the plane-strain RPV model and from the 3-D RPV model for: (a) point B; and (b) point C; along the curved crack front.

2) Post-process stress fields computed for the 2-D RPV model at each time increment over the loading history. Compute the Weibull stress values for each tip of the embedded crack and then calculate the constraint correction function $g(M, t, m)$ for each crack tip over the transient history $(t)$ using,

$$
g(M, t, m)=\frac{\sigma_{w, 2-D R P V}^{m}}{\tilde{C} L_{2-D, R P V} K_{J, 2-D R P V}^{4}}
$$

Here, the nonlinear $K_{J}$-values are those computed at crack tips in the 2-D RPV model under the pressure-thermal transient. $L_{2-D, R P V}$ denotes the length of the crack front in the finite element mesh for the 2-D, RPV model (often $L_{2-D, R P V}=1$ in FE models using 2-D elements).

3) The "local" Weibull stress values at the corresponding crack-front location of the 3-D RPV model over the transient history, therefore has the form,

$$
\sigma_{w, 3-D R P V}^{m}=\tilde{C} L_{3-D, R P V} K_{J, 3-D R P V}^{4} g(M, t, m)
$$


Here, $L_{3-D, R P V}$ denotes the length of the crack segment of interest located at some position on the 3-D crack front.

4) Repeat the above steps to compute Weibull stress values at other locations along the 3-D crack front that also have negative $\beta$-values.

Figure 14 compares the Weibull stress values estimated via Eqs. 13-15 using (a) the constraint function, $g(m)$, derived as described here from the 2-D RPV model and (b) 3-D $K_{J}$-values with the Weibull stress values computed from the full 3-D RPV model for four crack-front locations B, C, D and E. Two different plane-strain RPV models are used corresponding to the slightly different crack lengths from locations $\mathrm{B}$ to $\mathrm{C}$ and from $\mathrm{D}$ to $\mathrm{E}$. To construct these comparisons, the thicknesses specified for the two 2D RPV models equal the length of the crack-front segment at points B and C, and D and E (i.e., $\left.L_{2-D, R P V}=L_{3 D, R P V}\right)$, respectively. The Weibull stresses calculated from the constraint correction function obtained from the 2-D RPV model provide highly accurate estimates for the $\sigma_{w}$-values both for relatively straight crack-front locations (B and C) and for more strongly curved crack-front locations (D and E).
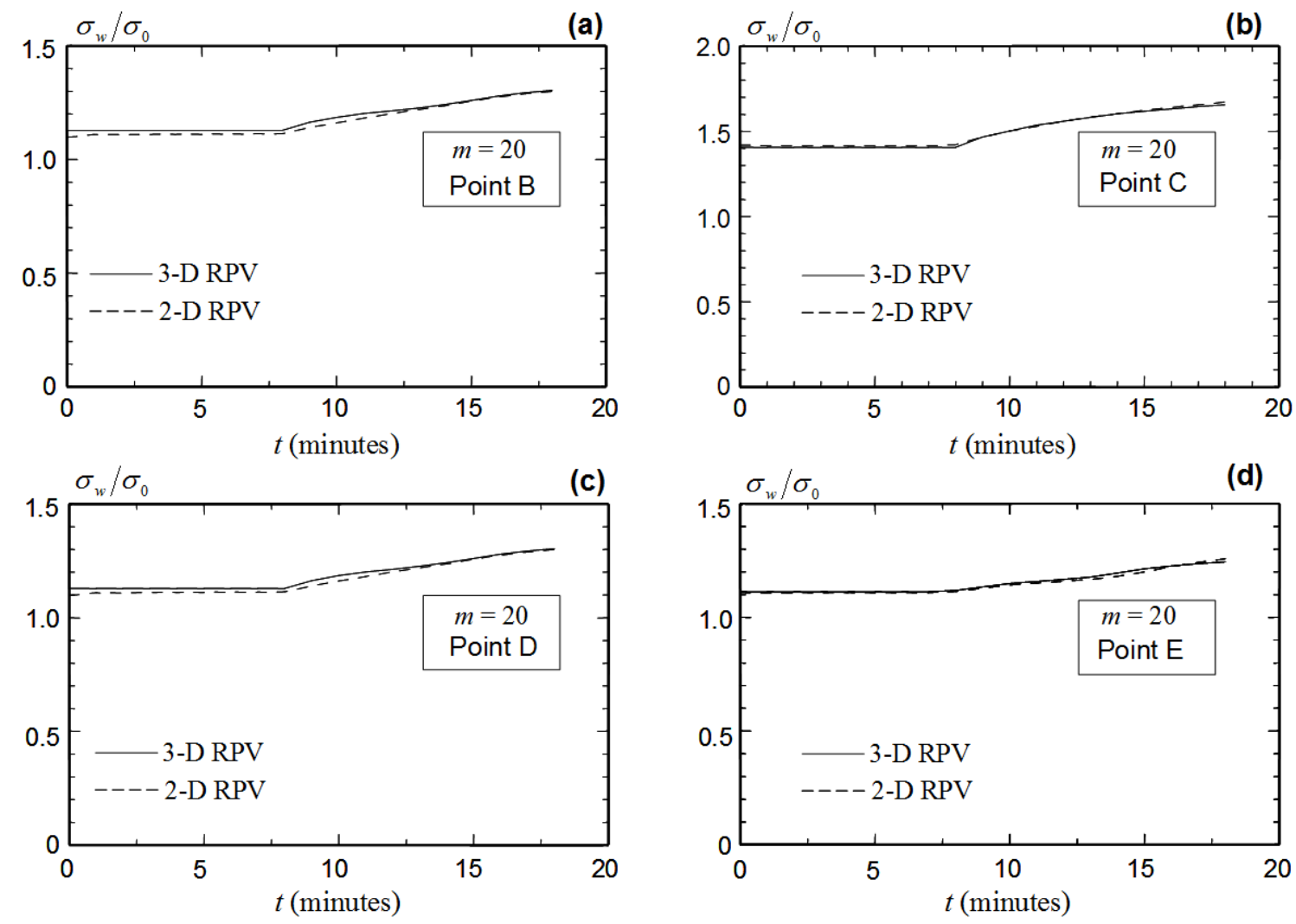

Figure 14 Evolution of the Weibull stress over the transient history computed from the plane-strain RPV model and from the 3-D RPV model for: (a) point B; (b) point C; (c) point D; and (d) point $\mathrm{E}$; along the curved crack front. 
For locations with high constraint $(\beta \geq 0)$ along the 3-D crack front, the conventional MBL model proves accurate for computation of Weibull stress values. The MBL model is subjected to the combination of $K_{I}$ and $T$-stress loading generated by the $\beta$-value at the location of interest on the 3-D crack front. Using $\tilde{C}$ defined in Eq. 13 obtained from the boundary layer analysis, the Weibull stress at a crack front location in the 3-D RPV model follows as,

$$
\sigma_{w, 3-D R P V}^{m}=\tilde{C} L_{3-D, R P V} K_{J, 3-D R P V}^{4}
$$

where $L_{3-D, R P V}$ denotes the length of the crack-front segment of interest at the high-constraint location. Here also, $K_{J}$ defines the local value of the elastic-plastic, crack-front driving force. Figure 15 demonstrates the excellent agreement between the Weibull stress value obtained from the plane-strain MBL model and that near point A in the 3-D RPV model.
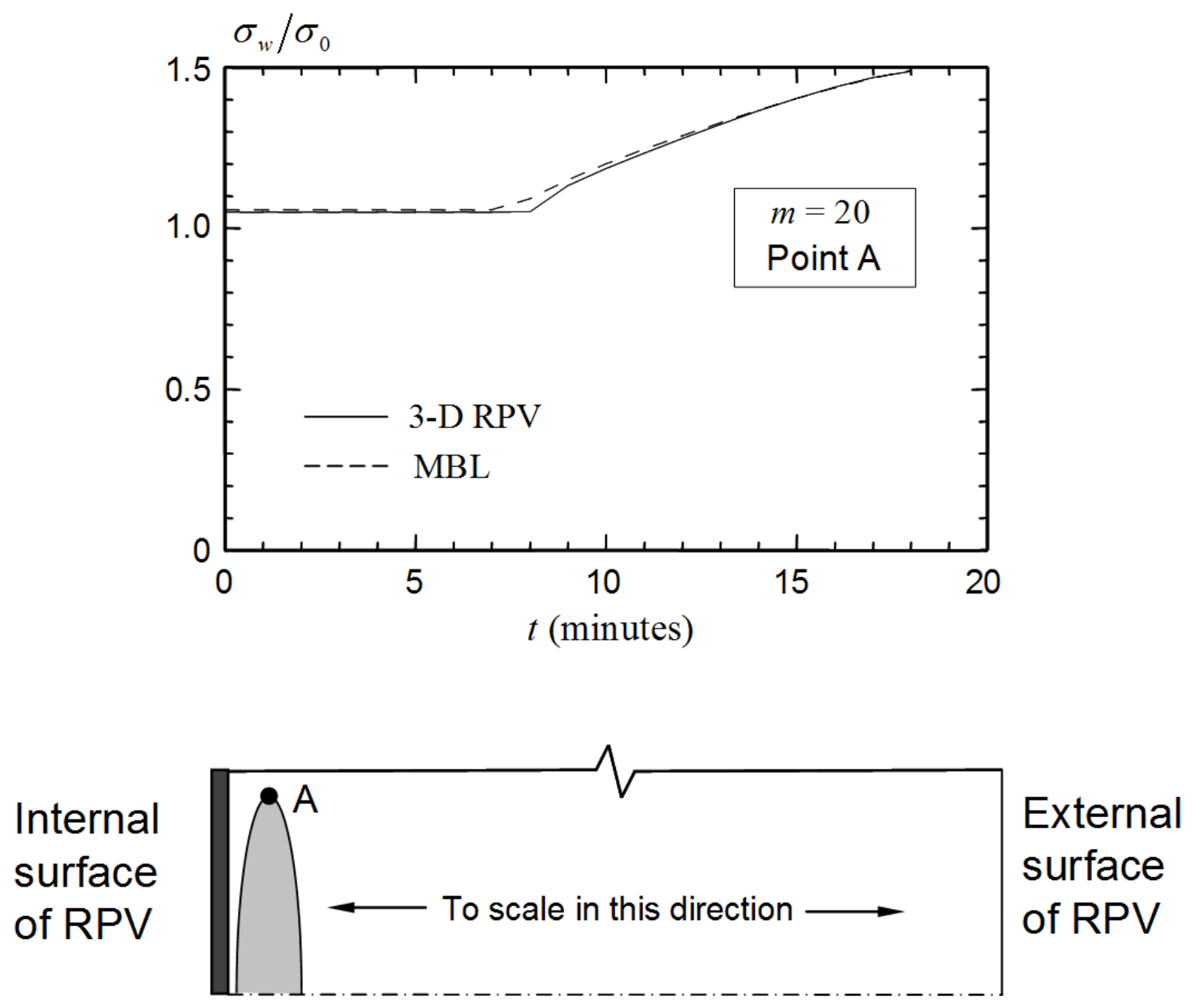

Figure 15 Evolution of the Weibull stress over the transient history computed from the plane-strain 2-D RPV model and from the 3-D RPV model for point $A$ along the curved crack front. 
For crack-front locations (points B, C, D and E) with low constraint in the 3-D RPV model, the Weibull stress computed from the 2-D RPV model at $t=18$ min achieves greater than $99 \%$ accuracy compared to the Weibull stress values computed from the 3-D RPV model. For crack-front locations (point A) with relatively high constraint, the difference in the Weibull stress values at $t=18$ min computed from Eq. 16 and from the 3-D RPV model is less than 1\%.

The computation of Weibull stress values for the 3-D crack-front locations with very low constraint utilizes the $g(m)$ function derived from 2-D RPV models, see Eq. 14. This procedure requires specification of the elastic-plastic $K_{J}$-value and a crack-front length for the actual 3-D crack-front geometry. The $\sigma_{w}^{m}$-value in Eq. 15 has a linear relationship with the crack-front length, which affects the volume of the fracture process zone. The accuracy of the pointwise Weibull stress values for the 3-D RPV crack front increases with the number of crack-front segments that are approximated using the 2-D RPV model. Approximate procedures may divide the entire elliptical crack front (half of the elliptical crack front is shown in Fig. 2) into four segments: two segments centered over points B and C with low constraint (negative $T$-stresses) and two segments centered over the two ends of the ellipse, e.g., point A, with high constraint (positive $T$-stresses). Consequently, the assessment procedure computes the cumulative probability of fracture using four values of the local Weibull stress along the entire crack front, following Eq. 7. The Weibull stress value (Eq. 15) for the low-constraint, crack-front region adopts a value of $L_{3-D, R P V}$ equal to the length for the relatively straight crack front, e.g., 0.8(2c), with $c$ shown in Fig. 2. With a selected (or unit) thickness of the 2-D RPV model, the 3-D Weibull stress for the relatively straight crack front becomes,

$$
\sigma_{w, 3-D R P V}^{m}=\sigma_{w, 2-D R P V}^{m} \frac{L_{3-D R P V}}{L_{2-D R P V}}\left(\frac{K_{J, 3-D R P V}}{K_{J, 2-D R P V}}\right)^{4}
$$

The elastic-plastic $K_{J}$-values have the maximum value in each crack-front segment corresponding to the largest crack depth, e.g., point B for the crack-front segment near the inner surface of the RPV and point C for the crack-front segment near the outer surface. These $K_{J}$-values yield an estimated Weibull stress value larger than the local Weibull stresses computed from a detailed 3-D RPV model, and leads to a conservative prediction of the cumulative probability of fracture. The Weibull stress value for the two (more curved) crack-front segments with a positive $T$-stress derives from the boundary layer model subjected to $K_{I}$ only (zero $T$-stress) and a $L_{3-D, R P V}$ value equal to the length of the curved crack front, using Eq. 16. 
The Weibull scaling parameter, $\sigma_{u}$, in each crack-front segment follows from $\sigma_{u}=\sigma_{w}\left(K_{J}=K_{0}\right)$, where $K_{0}$ in each segment follows the Master Curve using temperature variations at points B, C and A [25]. The time-dependent temperature, defined by the thermal transient as a function of the radius of the RPV, does not vary significantly within the crack-front segment around point $\mathrm{B}$ or within that around point $\mathrm{C}$. The temperature at point A represents an average (temperature and therefore $\sigma_{u}$ ) value in the corresponding crack-front segment. The threshold Weibull stress value may take a temperature independent value derived from a constant $K_{\min }$ of $20 \mathrm{MPa} \sqrt{\mathrm{m}}$ as specified in E-1921.

\section{Summary and Conclusions}

This study investigates 2-D and 3-D, nonlinear modeling procedures for a planar, embedded flaw located in the wall of a reactor pressure vessel (RPV). The analyses provide mechanical response data (displacement, strain, stress) to support computation of the Weibull stress $\left(\sigma_{w}\right)$ values along the curved crack front for structural integrity assessments against cleavage fracture failure. The RPV considered here contains a credible, maximum size flaw idealized as an embedded ellipse having dimensions $2 a=17 \mathrm{~mm}$ and $a / c=0.17$, with the crack surface remaining parallel to the longitudinal axis of the RPV. The crack is located near the inside wall of the vessel adjacent to the austenitic steel cladding. The imposed pressure-thermal transient derives from simulations of a critical pressurized thermal shock event (PTS) triggered by a loss of coolant accident. The PTS transient causes significant overcooling initiated from the internal surface of the RPV, coupled with a pronounced reduction in the internal pressure at the initial stage of the transient. The exceptionally detailed, 3-D nonlinear analyses of the RPV provide benchmark values of the Weibull stress for assessment of three, simplified plane-strain models which include: the modified boundary layer model (MBL), the M(T) model and the 2-D RPV model. The simplified models will prove critical for computational practicality in expanded studies to consider the stochastic variability of flaw sizes, flaw location, material properties and thermo-mechanical loading.

The present study supports the following observations and conclusions:

(1) The thermal-mechanical transient imposed on the RPV generates significant variations in both the macroscopic crack driving force, $K_{J}$, and the microscopic crack driving force, $\sigma_{w}$, along the 3-D curved crack front. The location of the maximum $\sigma_{w}$-value does not correspond to the location of the maximum $K_{J}$-value due to significant variability in the plasticity driven constraint conditions along the crack front. Further complications will arise in applications from the temperature gradient 
over the wall thickness, which requires corresponding variations in the Weibull material parameters, $\sigma_{u}$ and $\sigma_{w-\min }$, along the crack front.

(2) The very detailed 3-D analyses to obtain Weibull stress values over the complete crack front reveal that only a few, critical segments along the crack front (away from the ends) actually contribute to the cumulative probability of cleavage fracture. Weibull stress (and $K_{J}$ ) values elsewhere fall near or below threshold levels or are small relative to peak values that act over larger volumes of material. These observations motivate the development of simplified 2-D models to compute the Weibull stress values that predict the same cumulative failure probabilities obtained from the full, 3-D analyses.

(3) For the crack size, shape, location and thermal-mechanical loading considered here, the T-stress remains quite negative ( $\beta<-0.5$ ) over the crack front except for very small portions at the two ends of the crack. For this embedded flaw, the modified boundary layer model (MBL) fails to correctly predict the effects of stress redistribution under increased plastic deformation in the same manner that it also fails to model a simple $\mathrm{M}(\mathrm{T})$ specimen. For far-field loading caused by internal pressure acting alone on the vessel, the crack-front opening mode stresses, $\sigma_{y y}$, computed from the MBL model fall increasingly below the values from the 3-D RPV model for increased loading levels above a normal operating pressure.

(4) A plane-strain model of an $\mathrm{M}(\mathrm{T})$ specimen, with a crack length $2 a$ that yields $\beta$ matching the RPV crack-front location and remote (uniform) tensile stress that matches the axial stress in the RPV from internal pressure, predicts very closely the crack-front stress, $\sigma_{y y}$, obtained in the 3-D detailed analyses. The agreement holds to internal pressures at least twice the normal operating pressure and applies at the most critical locations along the 3-D crack front. The M(T) model applies quite well since the $\beta$-value for the RPV and $\mathrm{M}(\mathrm{T})$ both remain equal and constant for no thermal transients. Addition of the thermal transient causes the RPV $\beta$-value to vary significantly over the loading history and invalidates the $\mathrm{M}(\mathrm{T})$ approximation for the combined thermal-mechanical loading.

(5) The plane-strain RPV model represents very accurately the evolution of plasticity-driven constraint conditions predicted by the 3-D RPV model over the combined thermal-pressure transient. The crack-front opening stress, $\sigma_{y y}$, shows excellent agreement with values computed near the corresponding crack-front locations in a 3-D RPV model. This model represents only a marginal increase in complexity and computational effort compared to the $\mathrm{M}(\mathrm{T})$ approximation. 
(6) For an RPV subjected only to internal pressure loading, highly accurate Weibull stress values are obtained from the $\mathrm{M}(\mathrm{T})$ approximation via Eq. 6. The constraint correction function, $g(M)$, follows from the $\mathrm{M}(\mathrm{T})$ model subjected to same $K_{J}$ and $T$-stress as the corresponding crack front location in the 3-D RPV. For an RPV subjected to a thermal-pressure transient, highly accurate Weibull stress values are obtained using the $g(M)$ function derived from the 2-D RPV model and the elastic-plastic $K_{J}$-value obtained from the 3-D RPV model.

(7) For pressure-thermal loading, the conventional MBL model proves very applicable to compute Weibull stress values at 3-D crack front locations having a positive $T$-stress.

\section{Acknowledgement}

This investigation was supported by grants principally from the U.S. Nuclear Regulatory Commission, Office of Regulatory Research and from the Naval Surface Warfare Center, Carderock Division. Additional financial support was provided by the M.T. Geoffrey Yeh Endowed Chair Fund in the Department of Civil \& Environmental Engineering at the University of Illinois. We wish to acknowledge the many valuable discussions and data contributions from Dr. Mark T. Kirk, Nuclear Regulatory Commission (NRC), and Mr. Charles Roe, Naval Surface Warfare Center (NSWC).

Notice: This manuscript has been authored by UT-Battelle, LLC, under contract DE-AC05-00OR22725 with the U.S. Department of Energy. The United States Government retains and the publisher, by accepting the article for publication, acknowledges that the United States Government retains a nonexclusive, paid-up, irrevocable, world-wide license to publish or reproduce the published form of this manuscript, or allow others to do so, for United States Government purposes.

\section{References}

1. Schuster, G. J., Doctor, S. R. and Heasler, P. G. (1998). Characterization of flaws in US reactor pressure vessels, density and distribution of flaw indications in PVRUF. NUREG/CR-671, Vol 1, PNNL-1 1143. US Nuclear Regulatory Commission, Washington, D. C.

2. Schuster, G. J., Doctor, S. R. and Heasler, P. G. (1999). Characterization of flaws in US reactor pressure vessels, density and distribution of flaw indications in the Shoreham vessel. NUREG/CR6471, Vol. 3, PNNL-1 1143, US Nuclear Regulatory Commission, Washington, D. C.

3. Jackson, D. A., Doctor, S. R., Schuster, G and Simonen, F. (2001). Developing a generalized flaw distribution for reactor pressure vessels. Nuc. Engng. Design, 208, 123-131. 
4. Williams, P. T., Dickson, T. L. and Yin, S. (2004). Fracture analysis of vessels-Oak Ridge FAVOR, v04.1, computer code: theory and implementation of algorithms, methods, and correlations. NUREG/CR-6854.

5. Dickson, T. L., Williams, P. T. and Yin, S. (2004). Fracture analysis of vessels-Oak Ridge FAVOR, v04.1, computer code: user's guide. NUREG/CR-6855.

6. Beremin, F. M. (1983). A local criterion for cleavage fracture of a nuclear pressure vessel steel. Metallur. Trans., 14(A), 2277-2287.

7. Mudry, F. (1987). A local approach to cleavage fracture. Nucl. Engng. Design, 105, 65-76.

8. Griffith, A. A. (1920). The phenomena of rupture and flow in solids. Phil. Trans., 221(A), 163-198.

9. Lidbury, D. P. G, Bass, B. R., Bhandari, S. and Sherry, A. H. (2001). Key features arising from structural analysis of the NESC-1 PTS benchmark experiment. Int. J. Pres. Ves. Piping, 78, 225236.

10. Bernauer, G., Brocks, W. and Schmitt, W. (1999). Modifications of the Beremin model for cleavage fracture in the transition region of a ferritic steel. Engng. Fract. Mech. 64, 305-325.

11. Gao, X., Ruggieri, C. and Dodds, R. H. Jr. (1998). Calibration of Weibull stress parameters using fracture toughness data. Int. J. Fract., 92, 175-200.

12. El-Shennawy, M., Minami, F., Toyoda, M. and Kajimoto, K. (1999). Crack-tip constraint activators and the application to plane-strain fracture toughness test: Proposal on small-size fracture toughness specimen. Engng. Fract. Mech. 63, 447-479.

13. Ruggieri, C. and Dodds, R. H. Jr. (1998). Numerical evaluation of probabilistic fracture parameters with WSTRESS. Engng. Comp, 15, 49:73.

14. Minami, F., Bruckner-Foit, A., Munz, D. and Trolldenier, B. (1992). Estimation procedure for the Weibull parameters used in the local approach. Int. J. Fract. 54, 197-210.

15. Gao, X. and Dodds, R. H. Jr. (2000). Constraint effects on the ductile-to-brittle transitions temperature of ferritic steels: a Weibull stress model. Int. J. Fract., 102, 43-69.

16. Ruggieri, C. and Dodds, R. H. Jr. (1996). Probabilistic modeling of brittle fracture including 3-D effects on constraint loss and ductile tearing. J. Phys. IV, 6(C6), 353-62.

17. Petti, J. P. and Dodds, R. H. Jr. (2002). Constraint comparisons for common fracture specimens: C(T)s and SE(B)s. Engng. Fract. Mech., 71, 2677-2683.

18. Petti, J. P. and Dodds, R. H. Jr. (2004). Coupling of the Weibull stress model and macroscale models to predict cleavage fracture. Engng. Fract. Mech., 71, 2079:2103.

19. Test method for the determination of reference temperature $T_{0}$ for ferritic steels in the transition range (ASTM E1921), (2002). American Society for Testing and Materials, Philadelphia. 
20. Cravero, S. and Claudio, R. (2005). Correlation of fracture behavior in high pressure pipelines with axial flaws using constraint designed test specimens - Part I: Plane-strain analysis. Engng. Fract. Mech., 72, 1344-1360.

21. Silva, L. A. L., Cravero, S. and Ruggieri, C. (2006). Correlation of fracture behavior in high pressure pipelines with axial flaws using constraint designed test specimens. Part II: 3-D effects on constraint. Engng. Fract. Mech. 73, 2123-2138.

22. Wallin, K. (1984). The scatter in $K_{I C}$-results. Engng. Fract. Mech., 19, 1085-1093.

23. Wallin, K., Saario, T. and Torronen, K. (1984). Statistical model for carbide induced brittle fracture in steel. Metal Science, 18, 13-16.

24. Lin, T., Evan, A. G. and Ritchie, R. O. (1986). A statistical model of brittle fracture by transgranular cleavage. J. Mech. Phys. Solids. 21, 263-277.

25. Petti, J. P. and Dodds, R. H. Jr. (2005). Calibration of the Weibull stress scale parameter, $\sigma_{u}$, using the Master Curve. Engng. Fract. Mech., 72, 91-120.

26. Wasiluk, B., Petti, J. P. and Dodds, R. H. Jr. (2006). Temperature dependence of Weibull stress parameters: studies using Euro-material. Engng. Fract. Mech., 73, 1046-1069.

27. Heerens, J. and Hellmann, D. (2002). Development of the Euro fracture toughness dataset. Engng. Fract. Mech., 69, 421-449.

28. Wallin, K. (2002). Master curve analysis of the Euro fracture toughness dataset. Engng. Fract. Mech. 69, 451-481.

29. Wasiluk, B., Qian, X., ORNL and Dodds, R. H., Jr. (2006). Nonlinear analyses for embedded cracks under pressurized thermal shock: comparisons with FAVOR and Weibull stress approaches. NUREG Report.

30. Yin, S., Bass, B. R., Williams, P. T. and Dickson, T. L. (2005). Develop benchmark problem for Tstress assessment of embedded flaw. ORNL Report.

31. Arcieri, W. C., Beaton, R. M., Fletcher, C. D. and Bessette, D. E. (2003). RELAP5 thermal hydraulic analysis to support PTS evaluation for the Oconee-1, Beaver Balley-1, and Palisades nuclear power plants. NUREG report.

32. Structural Reliability Technology. (2005). FEACrack: 3-D finite element software for cracks, version 2.7: user's manual.

33. Gullerud, A., Koppenhoefer, K., Roy, A., RoyChowdhury, S., Walters, M., Bichon, B., Cochran, K., Carlyle, A., and Dodds, R. H. Jr. (2006). WARP3D: 3-D dynamic nonlinear fracture analysis of solids using parallel computers and workstations. Structural Research Series (SRS) 607, UILUENG-95-2012, University of Illinois at Urbana Champaign. 
34. Walter, M., Paulino, G. and Dodds, R. H. Jr. (2005). Interaction-integral procedures for 3-D curved cracks including surface tractions. Engng. Fract. Mech., 72, 1635-1663.

35. Williams, M. L. (1957). On the stress distribution at the base of a stationary crack. ASME J. Applied Mech., 24, 109-114.

36. Shih, C. F. (1981). Relationships between the $J$-integral and the crack opening displacement for stationary and extending cracks. J. Mech. Phy. Solids. 29, 305-326.

37. Wang, Y. Y. and Parks, D. M. (1995). Limits of $J$-T characterization of elastic-plastic crack-tip fields. Constraint Effects in Fracture Theory and Applications: Second Volume, ASTM STP, 1244, 43-67. 